
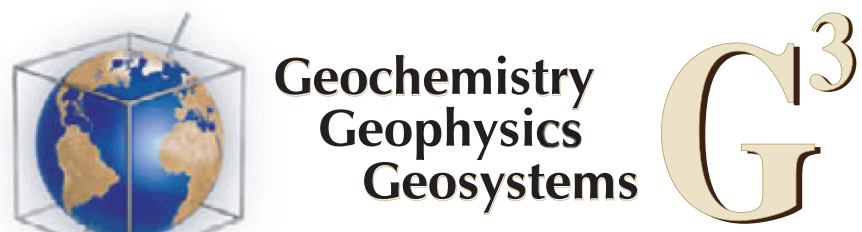

\title{
A paleointensity technique for multidomain igneous rocks
}

\author{
Huapei Wang \\ Earth and Planetary Sciences, Rutgers University, Piscataway, New Jersey,08854, USA (huapei@rci.rutgers.edu) \\ Dennis V. Kent \\ Earth and Planetary Sciences, Rutgers University, Piscataway, New Jersey, 08854, USA
}

Lamont-Doherty Earth Observatory, Columbia University, Palisades, New York, 10964, USA

[1] We developed a paleointensity technique to account for concave-up Arai diagrams due to multidomain (MD) contributions to determine unbiased paleointensities for 24 trial samples from site GA-X in Pleistocene lavas from Floreana Island, Galapagos Archipelago. The main magnetization carrier is fine-grained low-titanium magnetite of variable grain size. We used a comprehensive back-zero-forth (BZF) heating technique by adding an additional zero-field heating between the Thellier two opposite infield heating steps in order to estimate paleointensities in various standard protocols and provide internal self-consistency checks. After the first BZF experiment, we gave each sample a total thermal remanent magnetization (tTRM) by cooling from the Curie point in the presence of a low (15 $\mu \mathrm{T})$ laboratoryapplied field. Then we repeated the BZF protocol, with the laboratory-applied tTRM as a synthetic natural remanent magnetization (NRM), using the same laboratory-applied field and temperature steps to obtain the synthetic Arai signatures, which should only represent the domain-state dependent properties of the samples. We corrected the original Arai diagrams from the first BZF experiment by using the Arai signatures from the repeated BZF experiment, which neutralizes the typical MD concave-up effect. Eleven samples meet the Arai diagram post-selection criteria and provide qualified paleointensity estimates with a mean value for site GA-X of $4.23 \pm 1.29 \mu \mathrm{T}$, consistent with an excursional geomagnetic field direction reported for this site.

Components: 10,313 words, 13 figures, 3 tables.

Keywords: absolute paleointensity; multidomain magnetite

Index Terms: 1521 Paleointensity: Geomagnetism and Paleomagnetism.

Received 15 May 2013; Revised 5 August 2013; Accepted 7 August 2013; Published 16 October 2013.

Wang, H., and D. V. Kent (2013), A paleointensity technique for multidomain igneous rocks, Geochem. Geophys. Geosyst., 14, 4195-4213, doi:10.1002/ggge.20248.

\section{Introduction}

[2] Various techniques have been developed to determine the intensity of Earth's ancient magnetic field (paleointensity). The earliest double heating methods, which compare the incremental demag- netization of the natural remanent magnetization (NRM) and laboratory-acquired thermal remanent magnetization (TRM) to simulate the original remanence acquisition processes, developed by Thellier and Thellier [1959] and later modified by Coe [1967] and Aitken et al. [1988], still provide the 
most reliable paleointensity estimations. However, TRM theory [Neel, 1951] is only strictly applicable to stable single-domain (SSD) particles, which are expected to meet the requirements of the three Thellier laws (additivity, reciprocity, and independence of partial TRM (pTRM) [Thellier, 1938]). The grain size range for SSD magnetite, an ideal remanence carrier, is very narrow, usually only between about 30 and $200 \mathrm{~nm}$ at room temperature depending on grain shape [Butler and Banerjee, 1975]. Therefore, magnetite grains in even rapidly cooled volcanic rocks tend to have grain size distributions that extend into the multidomain (MD) range, where a single ferrimagnetic crystal is naturally divided into multiple magnetic domains separated by domain walls. Even for those materials that are thought to contain mainly SSD magnetite, such as submarine basaltic glass [Tauxe and Love, 2003] and copper slag [Ben-Yosef et al., 2008], it is often difficult to exclude the presence of some larger yet volumetrically significant MD grains. MD (titano)magnetite is expected to be an important contributor to the magnetization of most igneous rocks. Therefore, developing techniques to acquire reliable paleointensities from such material is critical for further analyses of the paleomagnetic field.

[3] The problem of trying to obtain reliable paleointensity estimates from samples that contain MD magnetite grains has been described by Levi [1977]. He concluded that paleointensities might be over-estimated due to the concave-up Arai diagram [Nagata et al., 1963] and the tendency to use the lower temperature segment of the NRMpTRM curve to calculate paleointensity to avoid complications due to thermochemical alterations. $X u$ and Dunlop [2004] theoretically and experimentally studied the shapes of Arai diagrams for sized SSD and MD magnetite assemblages and found that the larger the MD particles are, the greater the curvature of the Arai diagram is, which eventually approaches the curve predicted by MD field blocking theory. Nevertheless, both studies found that the beginning and end points of the Arai diagram are not affected by the concave shape of the curve, which indicates that the total TRM (tTRM) is reproducible.

[4] Shcherbakov et al. [1993] and Shcherbakova et al. [2000] studied the properties of pTRM of MD magnetite grains within natural and synthetic samples. They found that the Thellier laws of additivity and independence are violated in MD grains in the process of Thellier series paleointensity experiments. Dunlop and Ozdemir [2000] and $\mathrm{Fa}$ bian [2000, 2001] studied the blocking temperatures $\left(\mathrm{T}_{\mathrm{b}}\right)$ and the unblocking temperatures $\left(\mathrm{T}_{\mathrm{ub}}\right)$ of magnetite particles of various grain size ranges. They found that the concave-up Arai diagram for MD magnetite samples are exclusively due to the fact that their $\mathrm{T}_{\mathrm{ub}}<\mathrm{T}_{\mathrm{b}}$.

[5] Previous work has attempted to detect nonlinear MD behavior in paleointensity experiments by inserting additional heating steps into the Coe [1967] protocol [Riisager and Riisager, 2001] or by analyzing Arai diagram curvatures [Paterson, 2011]. The objective is to provide sufficient criteria to exclude non-ideal MD paleointensities, leaving only the contribution from specimens with well-behaved SSD grains. Other attempts to improve the Thellier series experiment protocols, for instance the IZZI (alternating between Infield + Zero-field and Zero-field + In-field heating steps) method [Tauxe and Staudigel, 2004; Yu et al., 2004], are mainly designed to detect non-reciprocity in the paleointensity data. Biggin and Thomas [2003], Fabian [2001], and Leonhardt et al. [2004] discussed the use of pTRM checks in MD samples within the paleointensity experiments. Biggin and Boehnel [2003] and Fabian and Shcherbakov [2004] discussed possible effects of repeated heating during lab procedure. Leonhardt et al. [2004] and Paterson [2013] discussed the effects of anisotropy of remanent magnetization carriers on absolute paleointensity results. However, due to the threshold nature of these criteria, the final average paleointentisy results may still be biased even after the typical exclusion of most specimens in a study.

[6] Wilson [1961, 1962] developed a paleointensity method that compares the continuous thermal demagnetization curve of NRM to that of a laboratory-applied tTRM. The Wilson method is domain-status independent because it is comparing the magnetization unblocking spectra of the NRM and laboratory-applied tTRM. However, due to the fact that it measures a sample's magnetization at elevated temperature and provides no means to monitor thermal alteration, the Wilson method is not widely used.

[7] A previous theoretical model [Fabian, 2001] suggested an extended treatment for Thellier series paleointensity experiments, in which the tTRM produced after completion of the original Thellier experiment is stepwise thermally demagnetized. Fabian [2001] also suggested that if a specimen did not experience alteration during heating, accurate paleointensity could be estimated by plotting 


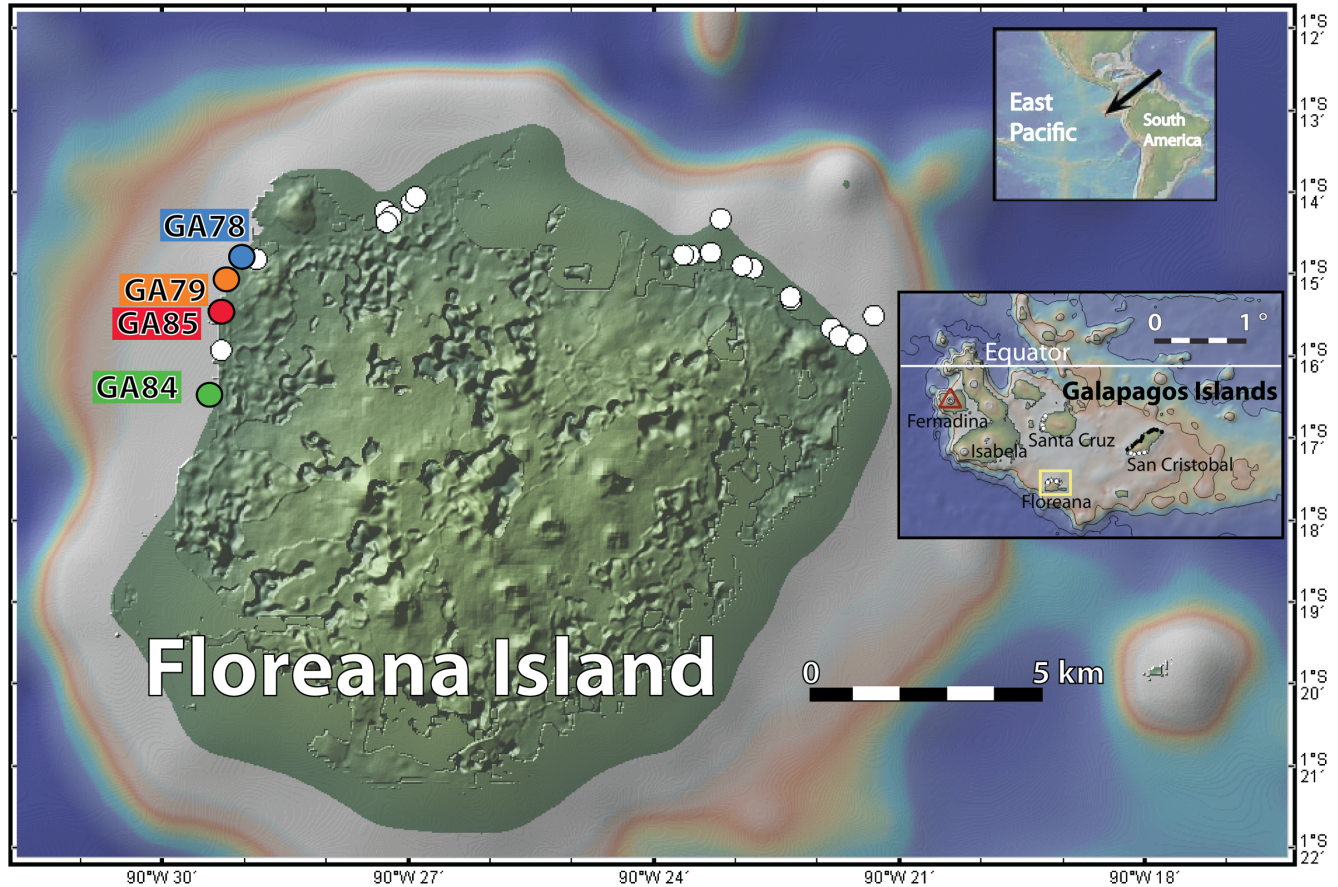

Figure 1. Location map of sites GA78 (blue), GA79 (orange), GA84 (green) and GA85 (red) from Floreana, Galapagos Islands. White dots are sampling sites from Rochette et al. [1997] that are not discussed in this paper. The map was generated by GeoMapApp using the Global Multi-Resolution Topography data (GMRT version 2.4)

its stepwise NRM losses versus tTRM losses (ideal pTRM) from a higher temperature range that avoided viscous remanent magnetization (VRM).

[8] In this study, we develop a Thellier series paleointensity technique that can not only detect the presence of MD remanence carriers but also provide a method to correct concave-up Arai diagrams to obtain unbiased paleointensity determinations. We selected 24 basaltic lava specimens from four sites (GA78, 79, 84, 85) from Floreana Island, Galapagos Archipelago, for detailed study. These sites were part of an extensive study of paleosecular variation for 0-3 Ma based on more than 50 sites from various Galapagos islands [Kent et al., 2010; Rochette et al., 1997]. The four sites, which we refer to collectively as locality GA-X, had virtually the same mean paleomagnetic direction that was moreover widely divergent from the overall mean bipolar directional axis. Thus, the four sites from the GA-X sampling locality are thought to represent the same short time interval. We expect to process the rest of the Galapagos collection of more than 300 samples to derive a time-averaged paleointensity value. Site GA-X simply provided a large number of individually oriented samples to develop and test a paleointensity technique that can be applied to the rest of the Galapagos sample collection. The GA-X samples had some of the lowest preliminary paleointensity values from the studied Galapagos lavas, and only a small fraction of today's field strength [Kent et al., 2010] and should, thus, provide a severe test of the efficacy of a paleointensity technique in the face of relatively larger effects of magnetic overprinting (VRM) and other secondary processes including laboratory-induced artifacts. Moreover, the divergent mean characteristic paleomagnetic direction allowed any VRM acquisition in the present-day field to be readily detected and discounted.

\section{Samples}

[9] The Galapagos Archipelago consists of volcanic islands on the Nazca plate just south of the Equator (Figure 1). The islands formed over a period of several million years, during which the Nazca plate moved east-southeast relative to the presumed Galapagos hotspot. Floreana Island (yellow square in Figure 1 inset), from which our samples were collected, is about $160 \mathrm{~km}$ to the southeast of the current eruptive center on Fernandina Island (red triangle in Figure 1 inset, $0.37^{\circ} \mathrm{S}$, 


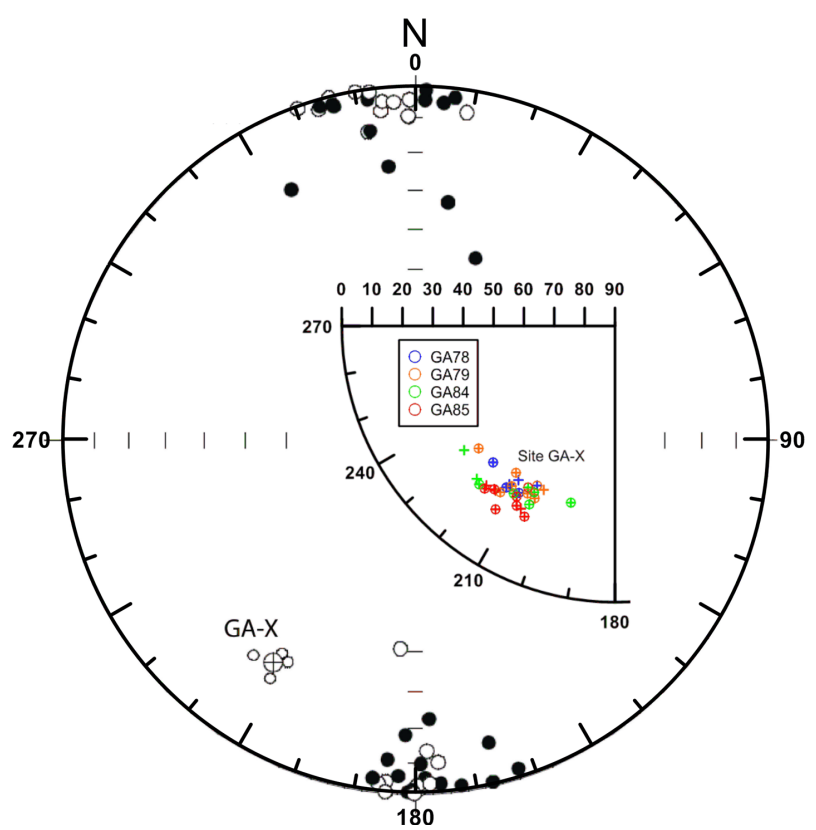

Figure 2. Filled and open circles are characteristic sitemean paleomagnetic directions plotted on an equal-area stereographic projection for all Galapagos sample sites (after Kent et al. [2010]). Colored symbols in the inset are paleomagnetic directions for samples from site GA-X (colored empty circles indicate samples used in previous paleo-directions studies; colored crosses indicate samples used in this paleointensity study).

$\left.91.55^{\circ} \mathrm{W}\right)$. The samples studied in this paper were taken with a hand-held gasoline-powered drill from four sites (GA78, GA79, GA84, and GA85) along the northwest coast of Floreana Island (Figure 1) on a 1993 expedition [Rochette et al., 1997]. Alternating field (AF) and thermal demagnetization (TD) analyses [Kent et al., 2010; Rochette et al., 1997] revealed that these four sites had virtually the same paleomagnetic directions (mean Decl $=212.7^{\circ}$; mean Incl $=-26.5^{\circ} ; \mathrm{A} 95=5.0^{\circ}$ ), which deviated by about $40^{\circ}$ from the average direction for all of the studied reverse polarity sites $\quad\left(\mathrm{N}=25 ; \quad \operatorname{Decl}=179.8^{\circ} ; \quad \operatorname{Incl}=-0.1^{\circ}\right.$; A95 $\left.=5.6^{\circ}\right)($ Figure 2). Paleointensities from these four sites (31 specimens) by a brute-force twopoint total TRM technique [Kent et al., 2010] yielded low values $($ mean $=5.7 \mu \mathrm{T}$, median $=4.4$ $\mu \mathrm{T}$; Table 1), less than $20 \%$ of today's equatorial dipole field. Judging from the coincidence of the paleomagnetic directions and paleointensities (Figure 2 and Table 1), as well as the close spatial proximity of these sites (Figure 1), we conclude that they are from essentially contemporaneous lava flows, if not from a single one. The samples can thus be combined to one site, GA-X, with not only the same paleomagnetic direction, but also the same expected paleointensity. The $25 \mathrm{~mm}$ diameter paleomagnetic core samples were sliced into several $12 \mathrm{~mm}$ height specimens, which were used in previous paleomagnetic directional studies ( $\mathrm{a}$ and b specimens) [Rochette et al., 1997; Kent et $a l ., 2010]$ and in this paleointensity study (c specimens). We also cut small ( $\sim 20 \mathrm{mg})$ chips directly from c specimens for rock magnetic studies in the hope of minimizing any mineralogical differences between paleointensity bulk specimens and the chips used for rock magnetic characterization. In total, data from 24 available c specimens from site GA-X were generated and analyzed in this study (Table 1).

\section{Rock Magnetic Experiments and Results}

\subsection{High-Temperature Magnetic Properties}

[10] We heated each of the 24 chip specimens to $600^{\circ} \mathrm{C}$ and cooled them back to room temperature at a rate of $50^{\circ} \mathrm{C} / \mathrm{min}$ on a Alpha Precision Instruments translation Curie balance at the Rutgers paleomagnetic laboratory in a $0.15 \mathrm{~T}$ field to measure their thermomagnetic properties as induced magnetization versus temperature $\left(\mathrm{J}_{\mathrm{s}}-\mathrm{T}\right)$ curves. We repeated the thermomagnetic experiments to check if the chips had been thermochemically altered, by comparing the $\mathrm{J}_{\mathrm{s}}-\mathrm{T}$ curves for the first and second heatings (Figures $3 \mathrm{a}-\mathrm{d}$ ). Chips that experienced little alteration should provide reversible first $\mathrm{J}_{\mathrm{s}}-\mathrm{T}$ curves and similar second $\mathrm{J}_{\mathrm{s}}-\mathrm{T}$ curves.

[11] First and second $\mathrm{J}_{\mathrm{s}}-\mathrm{T}$ curves for all 24 rock chips give Curie temperatures typically around between $550^{\circ} \mathrm{C}$ and $580^{\circ} \mathrm{C}$, which indicates that the major magnetization carrier is low-titanium magnetite. For almost all chips, heating and cooling curves for both the first and second Js-T experiments are similar, which indicates minimal thermochemical alteration in the course of the laboratory experiment (Figures 3a-d). According to the shape of the curves, we categorize the samples into two groups: Type I, in which the induced magnetization decreases slowly at low temperatures $\left(<400^{\circ} \mathrm{C}\right)$ and then decreases rapidly to the Curie point (see Figures $3 \mathrm{c}$ and d); and Type II, in which the induced magnetization decreases gradually over the entire temperature range to the Curie point (see Figures $3 a$ and $b$ ). Results for different $\mathrm{J}_{\mathrm{s}}-\mathrm{T}$ types are listed in Table 1. Most samples from 
Table 1. Samples Used in This Study and Their Rock Magnetic Properties ${ }^{\mathrm{a}}$

\begin{tabular}{|c|c|c|c|c|c|c|c|c|c|c|c|c|}
\hline Sample & $\begin{array}{l}\text { Used for } \\
\text { P-Int }\end{array}$ & $\operatorname{MAD}^{\mathrm{K}}\left(^{\circ}\right)$ & $\begin{array}{c}\text { Core PCA } \\
\text { Decl }^{\mathrm{K}} \\
\left({ }^{\circ}\right)\end{array}$ & $\begin{array}{c}\text { Core PCA } \\
\operatorname{Incl}^{\mathrm{K}} \\
\left({ }^{\circ}\right)\end{array}$ & $\begin{array}{c}\text { In Situ PCA } \\
\text { Decl }^{\mathrm{K}} \\
\left({ }^{\circ}\right)\end{array}$ & $\begin{array}{c}\text { In Situ PCA } \\
\operatorname{Incl}^{\mathrm{K}} \\
\left({ }^{\circ}\right)\end{array}$ & $\begin{array}{r}\mathrm{J}_{350-575}{ }^{\mathrm{K}} \\
\mathrm{E}-7\left(\mathrm{Am}^{2}\right)\end{array}$ & $\begin{array}{l}\text { Kent10 } \\
\text { P-Int }{ }^{\mathrm{K}} \\
(\mu \mathrm{T})\end{array}$ & $\begin{array}{l}\mathrm{J}_{\mathrm{s}}-\mathrm{T} \\
\text { Type }\end{array}$ & $\begin{array}{l}\mathrm{B}_{\mathrm{c}} \\
(\mathrm{mT})\end{array}$ & $\begin{array}{l}\mathrm{B}_{\mathrm{cr}} \\
(\mathrm{mT})\end{array}$ & $\begin{array}{r}\mathrm{M}_{\mathrm{r}} / \mathrm{M}_{\mathrm{s}} \\
\text { Ratio }\end{array}$ \\
\hline GA78.1 & Yes & 2.1 & 51.8 & -10.5 & 214.2 & -26.5 & 14.49 & 3.18 & I & 15.66 & 38.58 & 0.170 \\
\hline GA78.2 & Yes & 3.5 & 283.1 & -28.1 & 222.1 & -30.0 & 10.38 & 1.70 & II & 5.71 & 16.76 & 0.153 \\
\hline GA78.3 & No & 1.6 & 282.0 & -25.3 & 213.9 & -27.8 & 37.06 & 7.05 & N/A & N/A & N/A & N/A \\
\hline GA78.4 & No & 1.0 & 291.1 & -22.9 & 212.4 & -30.5 & 56.29 & 18.33 & N/A & N/A & N/A & N/A \\
\hline GA78.5 & Yes & 2.5 & 266.2 & -29.5 & 210.1 & -27.1 & 17.14 & 3.58 & I & 15.94 & 39.01 & 0.219 \\
\hline GA78.6 & Yes & 2.1 & 11.2 & -8.6 & 206.2 & -32.1 & 20.16 & 4.74 & I & 13.04 & 32.92 & 0.189 \\
\hline GA78.7 & No & 1.2 & 317.7 & -3.9 & 203.8 & -31.7 & 67.98 & 26.09 & N/A & N/A & N/A & N/A \\
\hline GA78.8 & Yes & 0.6 & 294.6 & -21.9 & 214.3 & -32.2 & 35.15 & 8.14 & I & 14.57 & 36.70 & 0.161 \\
\hline GA79.1 & Yes & 2.7 & 21.9 & -4.3 & 228.4 & -30.0 & 26.52 & 4.10 & II & 5.20 & 18.43 & 0.111 \\
\hline GA79.2 & Yes & 2.9 & 298.4 & -18.6 & 205.2 & -28.0 & 26.52 & 3.14 & II & 6.19 & 18.96 & 0.121 \\
\hline GA79.3 & Yes & 2.2 & 50.9 & -11.4 & 214.8 & -23.9 & 23.80 & 2.97 & I & 6.18 & 18.01 & 0.168 \\
\hline GA79.4 & Yes & 1.4 & 97.2 & -31.1 & 213.1 & -27.6 & 30.33 & 4.98 & I & 12.75 & 38.45 & 0.204 \\
\hline GA79.5 & Yes & 1.5 & 8.7 & -3.6 & 207.9 & -28.3 & 42.41 & 5.90 & II & 6.62 & 23.34 & 0.122 \\
\hline GA79.7 & Yes & 2.1 & 288.4 & -24.0 & 206.1 & -29.7 & 66.38 & 8.15 & I & 11.55 & 30.90 & 0.163 \\
\hline GA79.8 & Yes & 2.8 & 294.6 & -24.3 & 194.2 & -30.6 & 30.30 & 4.07 & II & 6.46 & 21.97 & 0.121 \\
\hline GA84.1 & Yes & 1.6 & 57.8 & -18.1 & 211.5 & -26.0 & 34.46 & 4.12 & I & 20.00 & 48.21 & 0.285 \\
\hline GA84.2 & Yes & 1.9 & 155.9 & -35.6 & 205.9 & -25.4 & 27.71 & 2.80 & I & 9.59 & 25.27 & 0.199 \\
\hline GA84.3 & Yes & 1.4 & 107.0 & -26.9 & 220.9 & -21.7 & 33.90 & 3.67 & I & 18.13 & 44.51 & 0.265 \\
\hline GA84.4 & No & 2.0 & 22.5 & -1.4 & 222.4 & -22.5 & 19.66 & 4.69 & N/A & N/A & N/A & N/A \\
\hline GA84.5 & No & 3.6 & 70.4 & -20.5 & 230.8 & -25.9 & 34.66 & 4.18 & N/A & N/A & N/A & N/A \\
\hline GA84.6 & Yes & 1.3 & 323.0 & -20.0 & 208.6 & -30.1 & 50.37 & 6.73 & I & 19.21 & 45.46 & 0.255 \\
\hline GA84.7 & No & 1.3 & 89.2 & -23.6 & 219.1 & -22.9 & 27.11 & 3.08 & N/A & N/A & N/A & N/A \\
\hline GA84.8 & Yes & 1.6 & 63.8 & -18.0 & 216.7 & -23.6 & 32.10 & 3.95 & II & 7.10 & 18.56 & 0.196 \\
\hline GA85.1 & Yes & 0.7 & 68.7 & -13.0 & 213.3 & -18.5 & 35.21 & 5.70 & II & 4.99 & 15.57 & 0.143 \\
\hline GA85.2 & Yes & 1.2 & 18.7 & -10.4 & 218.9 & -21.8 & 32.96 & 4.88 & II & 7.02 & 19.79 & 0.180 \\
\hline GA85.3 & Yes & 0.7 & 202.3 & -41.9 & 208.9 & -23.1 & 35.78 & 5.30 & II & 8.50 & 26.16 & 0.173 \\
\hline GA85.4 & Yes & 1.1 & 204.3 & -43.6 & 205.6 & -21.2 & 34.46 & 4.17 & II & 8.78 & 22.93 & 0.210 \\
\hline GA85.5 & Yes & 1.6 & 354.8 & -7.6 & 210.2 & -25.6 & 34.86 & 4.70 & II & 8.51 & 24.88 & 0.178 \\
\hline GA85.7 & Yes & 2.6 & 114.7 & -38.6 & 216.3 & -23.4 & 19.54 & 2.63 & II & 7.36 & 25.69 & 0.135 \\
\hline GA85.8 & Yes & 0.9 & 176.1 & -33.8 & 207.5 & -22.9 & 31.16 & 4.69 & I & 12.74 & 37.76 & 0.205 \\
\hline
\end{tabular}

${ }^{\mathrm{a}} \mathrm{MAD}$ is the maximum angular deviation, Core (In Situ) PCA Decl and Incl are the declination and inclination in sample core (in-situ geographic) coordinate frame from principal component analysis [Kirschvink, 1980], $\mathrm{J}_{350-575}$ is the magnetization vector length from principal component analysis between $350^{\circ}$ and $575^{\circ} \mathrm{C}, \mathrm{J}_{\mathrm{s}}-\mathrm{T}$ is the high field magnetization versus temperature curve, Kent $10 \mathrm{P}$-Int is the two-point preliminary paleointensity estimation, $B_{c}$ is the coercivity, $B_{c r}$ is the remanent coercivity, $M_{r} / M_{s}$ is the saturation remanent magnetization to saturation magnetization ratio. Columns superscripted by " $\mathrm{K}$ " are data from Kent et al. [2010]. Those used in previous paleomagnetic direction studies but not in this paleointensity study are due to unavailability of "c" specimens. For $\mathrm{J}_{\mathrm{s}}$-T curves, Type-I for more SSD dominating specimens; type-II for more MD dominating specimens.

sites GA78 and GA84 have Type I $\mathrm{J}_{\mathrm{s}}$-T curves, whereas most samples from sites GA79 and GA85 have Type II $\mathrm{J}_{\mathrm{s}}-\mathrm{T}$ curves.

\subsection{Magnetic Hysteresis Loops}

[12] To check if the chips had been thermophysicochemically altered ("physico" referring to domain state or structure), we measured hysteresis loops, isothermal remanent magnetization (IRM) acquisition curves and back field direct current demagnetization (DCD) curves for each of the 24 chips before and after the first thermomagnetic $\left(\mathrm{J}_{\mathrm{s}}-\mathrm{T}\right)$ experiments, using a Princeton Measurement Corporation alternating gradient magnetometer (AGM) MicroMag2900 in a maximum field up to $1 \mathrm{~T}$ at the Rutgers paleomagnetic laboratory. Each of our rock magnetic chip specimens was visually aligned in the same direction for hysteresis measurements before and after heating to factor out any contribution to the hysteresis signal due to anisotropy, whose effects are not expected to be important in these basalts. Representative hysteresis loops, IRM, and DCD curves are shown in Figures $3 \mathrm{e}-\mathrm{h}$. Hysteresis properties (magnetic coercivity, $\mathrm{B}_{\mathrm{c}}$; remanent coercivity, $\mathrm{B}_{\mathrm{cr}}$; and the ratio of the remanent to the saturation magnetization, $\mathrm{M}_{\mathrm{r}} / \mathrm{M}_{\mathrm{S}}$ ) of the samples are also listed in Table 1.

[13] Samples with Type $\mathrm{I}_{\mathrm{s}}-\mathrm{T}$ curves tend to have higher remanent coercivities and $\mathrm{M}_{\mathrm{r}} / \mathrm{M}_{\mathrm{s}}$ ratios than samples with Type II $\mathrm{J}_{\mathrm{s}}-\mathrm{T}$ curves (Figure 3 and Table 1). Generally, samples from sites GA78 and GA84 have higher coercivities and remanent coercivities $\left(\mathrm{B}_{\mathrm{cr}} \sim 10-20 \mathrm{mT} ; \mathrm{B}_{\mathrm{cr}} \sim 30-45 \mathrm{mT}\right)$ and $\mathrm{M}_{\mathrm{r}} / \mathrm{M}_{\mathrm{S}}$ ratios $(\sim 0.15-0.30)$ than samples from sites GA79 and GA85 ( $\mathrm{B}_{\mathrm{cr}} \sim 5-10 \mathrm{mT} ; \mathrm{B}_{\mathrm{cr}} \sim 15-$ $25 \mathrm{mT}$ and $\left.\mathrm{M}_{\mathrm{r}} / \mathrm{M}_{\mathrm{s}} \sim 0.10-0.20\right)$.

[14] Hysteresis properties of the 24 GA-X chips are summarized in Figure 4 in a Day plot [Day et al., 1977], on which data tend to be distributed 

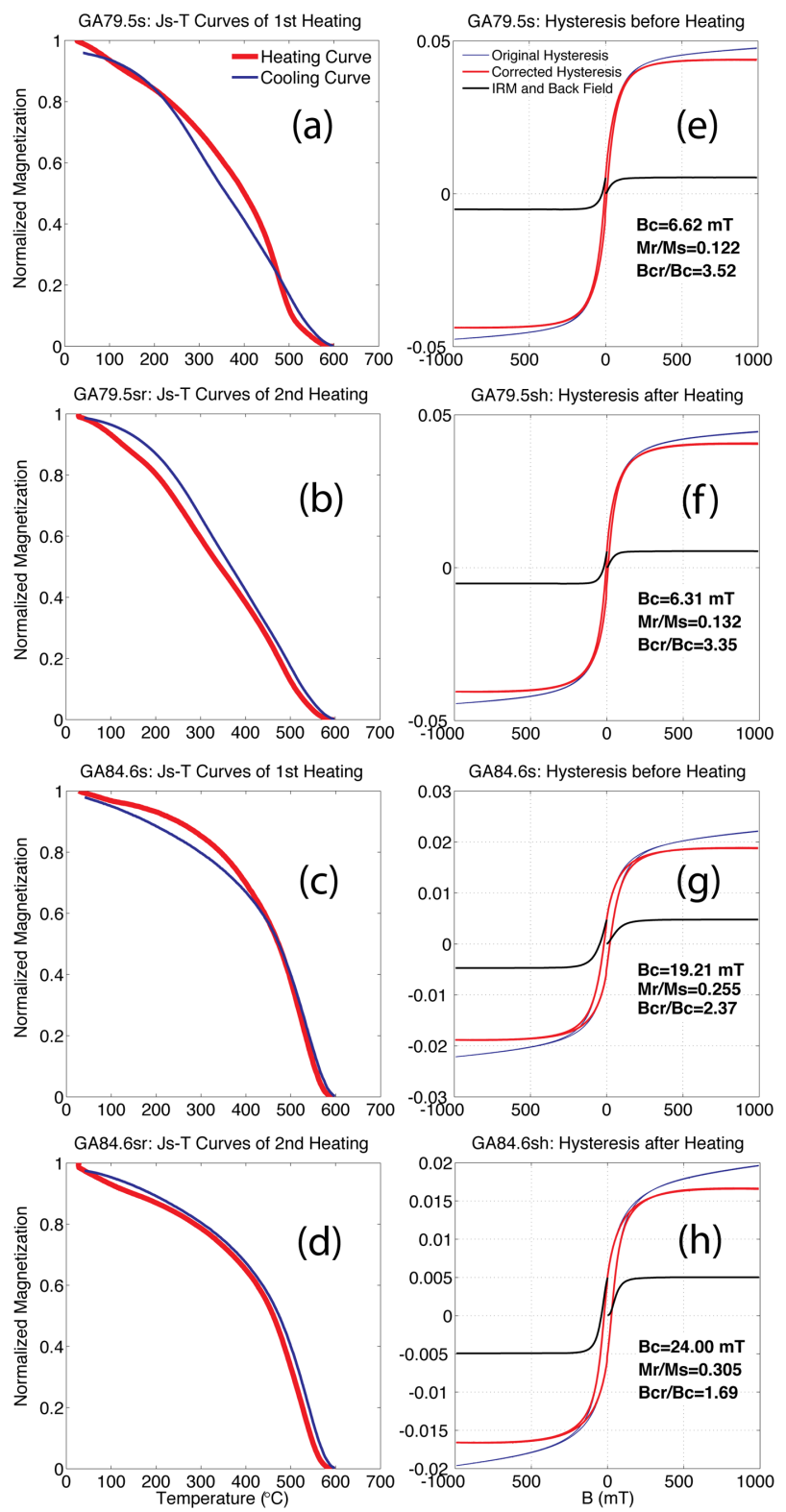

Figure 3. $\mathrm{J}_{\mathrm{s}}-\mathrm{T}$ curves for $(\mathrm{a}, \mathrm{c})$ the first and $(\mathrm{b}, \mathrm{d})$ second heating; hysteresis, IRM and back field DCD curves (e, g) before and $(f, h)$ after heating for specimens GA79.5s and GA84.6s.

along a theoretical SSD and MD mixing curve (\#3 from Dunlop and $X u$ [1994] and Xu and Dunlop [1994]). Samples from site GA79 have the highest concentration of MD grains of around $80 \%$ (by volume), followed by site GA85 and GA78 with around $70 \%$. Site GA84 has the lowest MD percentage of around $50 \%$.

[15] After heating to $600^{\circ} \mathrm{C}$, samples tend to move to the SSD corner of the Day plot, which indicates a reduction in the average effective grain size (increase of SSD domain state). The lower the MD

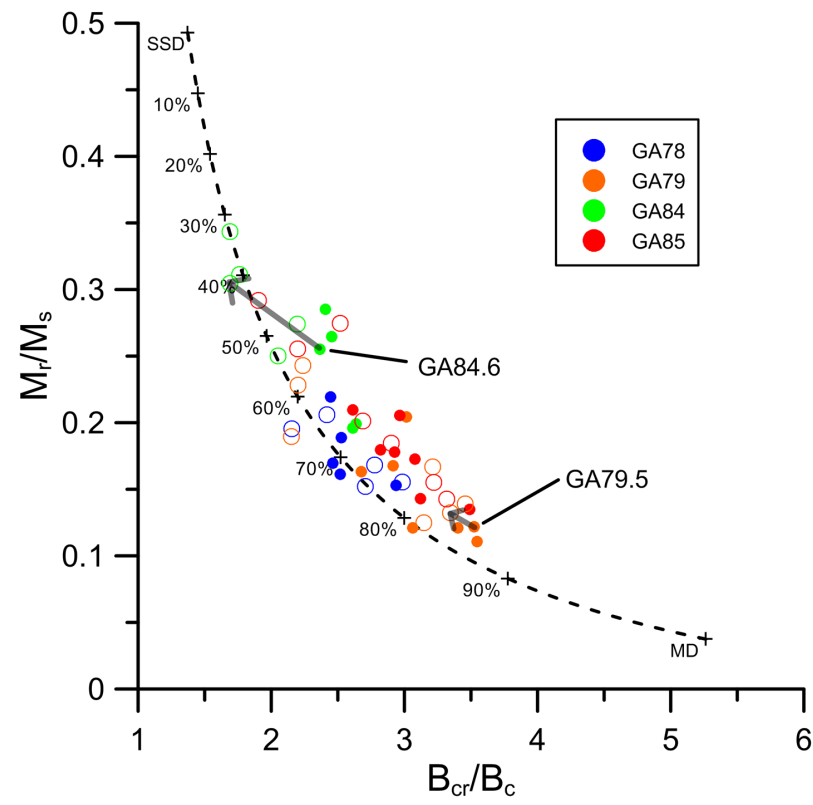

Figure 4. Day plot [Day et al., 1977] for GA-X samples. Dashed line is SSD-MD mixing curve 3 [Dunlop and $X u$, 1994; Xu and Dunlop, 1994], with crosses indicating the volume percentage of MD grains in the mixture. Filled circles are data before heating, and open circles are data after heating to $600^{\circ} \mathrm{C}$. Arrows indicate the alteration path for specimens GA79.5s and GA84.6s.

percentage (the smaller overall grain size) the sample initially had, the more severe the change it experienced due to heating. Samples from sites GA78, GA79, and GA85 experienced relatively less domain state changes, while the domain state of samples from site GA84 experienced greater changes (Figure 4).

[16] To summarize, rock magnetic properties of the 24 GA-X samples indicate that the dominant magnetic mineral is fine-grained, low-titanium magnetite with subequal SSD and MD-like contributions for sites GA78 and GA84 and with a more MD character for sites GA79 and GA85. Almost all of the samples are thermochemically stable even when heated to just above the Curie temperature of $580^{\circ} \mathrm{C}$. However, small but detectable thermophysical effective grain size (domain state) changes occurred during heating for the more SSD samples (i.e., those with the higher $\mathrm{M}_{\mathrm{r}} / \mathrm{M}_{\mathrm{S}}$ ratios), which are mainly from sites GA78 and GA84. The GA-X samples should thus be ideal candidates for Thellier paleointensity experiments in terms of their thermochemical stability, but less obviously so in terms of magnetic grain size, which tends to be dominated by MD carriers. 


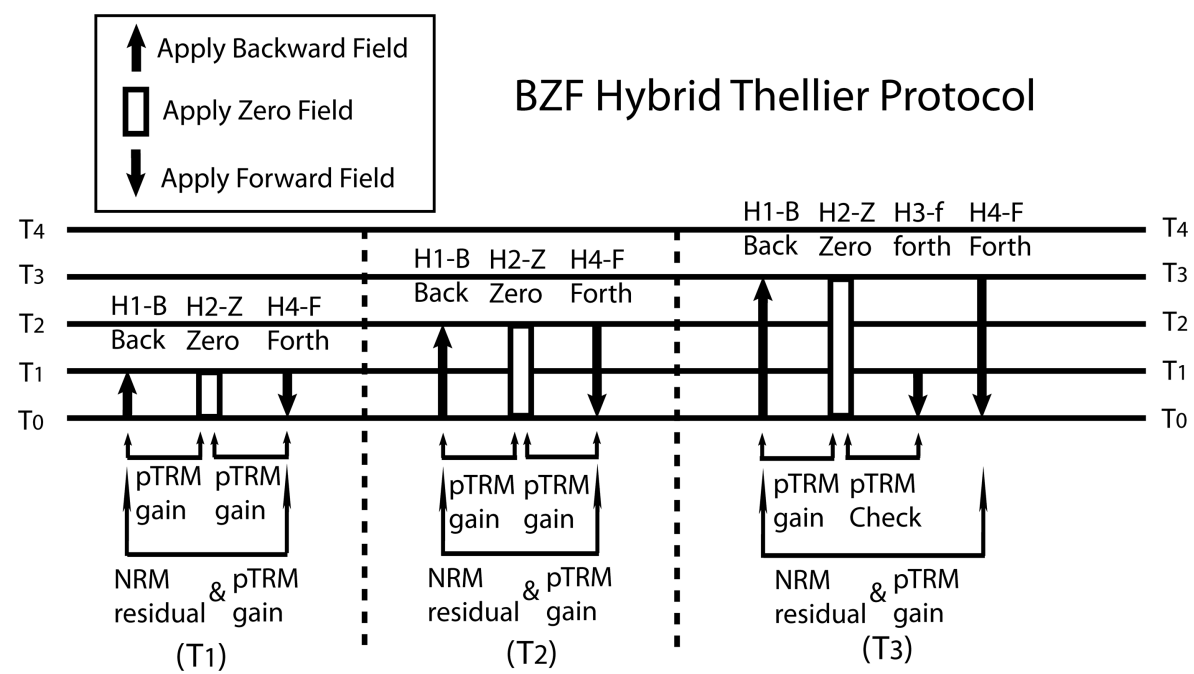

Figure 5. Schematic diagram with heating steps (H1-Backward field, H2-Zero field, H3-forward field to a previous lower temperature to perform pTRM check, H4-Forward field) for target temperatures (T1, T2, $\mathrm{T} 3, \ldots)$ in the proposed hybrid BZF protocol.

\section{Paleointensity Experiments and Results}

\subsection{Back-Zero-Forth (BZF) Protocol for Thellier Experiments and Results}

[17] There are three main double-heating paleointensity protocols: the classic Thellier method [Thellier and Thellier, 1959], and the Coe [1967] and Aitken et al. [1988] variants. Only the classical Thellier method is thought to be free from dependence on initial state [Yu and Tauxe, 2005]. In order to compare these paleointensity protocols for individual samples, we developed a hybrid tripleheating method, the BZF protocol, that consists of successive back-field heating, zero-field heating and forward-field heating. Samples were heated to target temperatures in zero-field and cooled to room temperature for the three BZF heating cycles per temperature step: the first cooling was performed in a $15 \mu \mathrm{T}$ laboratory-applied field (used throughout) along the sample $+\mathrm{Z}$ axis; the second cooling was performed in a zero-field environment; the third cooling was performed in the same $15 \mu \mathrm{T}$ laboratory-applied field but in the opposite direction (sample $-\mathrm{Z}$ axis) used for the first cooling. Partial TRM (pTRM) back checks were performed every other temperature step by reheating samples to lower temperatures in the same $15 \mu \mathrm{T}$ laboratory-applied field along the sample $-\mathrm{Z}$ axis after the second zero-field heating cycle (Figure 5). From room temperature, we used $100^{\circ} \mathrm{C}$, $200^{\circ} \mathrm{C}, 300^{\circ} \mathrm{C}, 350^{\circ} \mathrm{C}, 375^{\circ} \mathrm{C}, 400^{\circ} \mathrm{C}\left(350^{\circ} \mathrm{C}\right)$, $425^{\circ} \mathrm{C}, 450^{\circ} \mathrm{C}\left(400^{\circ} \mathrm{C}\right), 475^{\circ} \mathrm{C}, 500^{\circ} \mathrm{C}\left(450^{\circ} \mathrm{C}\right)$, $525^{\circ} \mathrm{C}, 550^{\circ} \mathrm{C}\left(500^{\circ} \mathrm{C}\right)$, and $575^{\circ} \mathrm{C}$ as the heating target temperatures (with pTRM back check heating temperatures in parentheses) for all 24 specimens. Combination of the first and third heating cycles corresponds to the original Thellier (two infield heating) protocol, combination of the second and third cycles corresponds to the Coe (zero-field and in-field) protocol, and combination of the first and second cycles corresponds to the Aitken (infield and zero-field) protocol. However, due to the MD high blocking temperature tails from the first heating of each temperature steps, which are conducted in a laboratory-applied back-field, the calculation of the Coe protocol in the BZF method is slightly different from the original Coe method. Hence, we note this calculation as the Coe* protocol.

[18] Besides testing the internal consistency of these three standard Thellier series protocols, the BZF protocol also allows paleointensities to be calculated from three extra NRM and pTRM combinations, due to the capability of the BZF protocol to calculate the NRM residual in two ways and the pTRM gain in three ways. However, we only used the outcomes calculated using the Thellier, $\mathrm{Coe}^{*}$ and Aitken protocols from the BZF experiments to estimate and compare paleointensity results. If the experimental conditions are ideal, the outcomes from the three classic methods (Thellier, Coe*, and Aitken) using the BZF protocol are expected to be identical for samples that contain only SSD particles, as predicted by Neel theory [Neel, 1951]. However, for samples 
NRM (*26.590 mA/m)

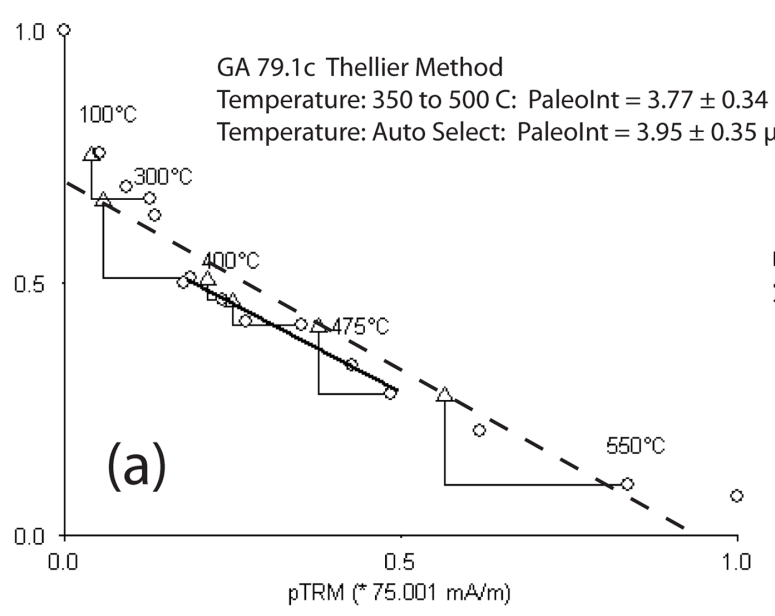

NRM (* $26.590 \mathrm{~mA} / \mathrm{m})$

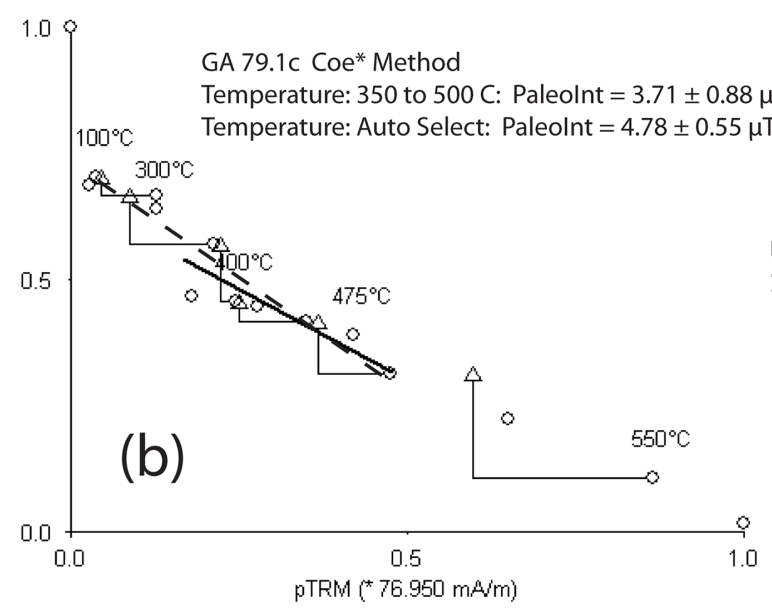

NRM (* $26.590 \mathrm{~mA} / \mathrm{m})$

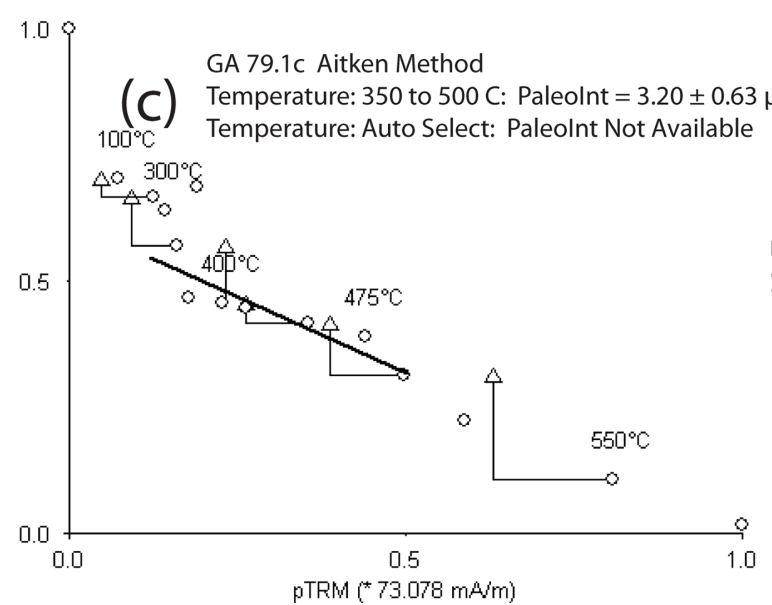

GA79.1c

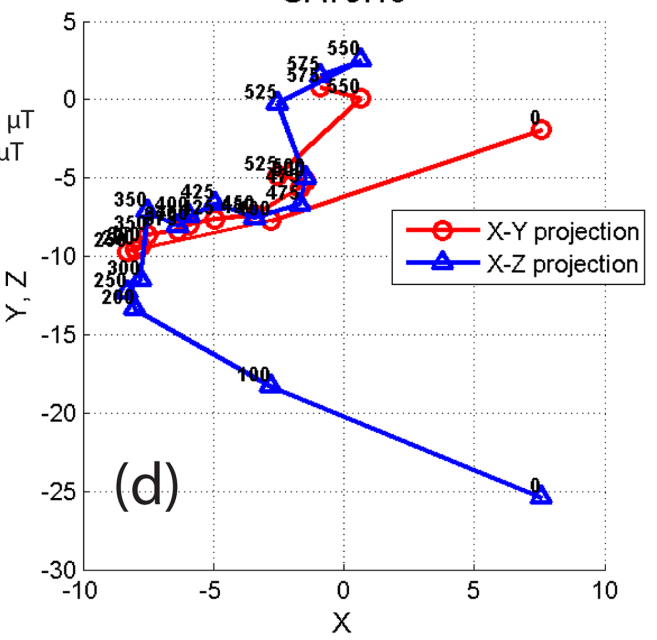

GA79.1c

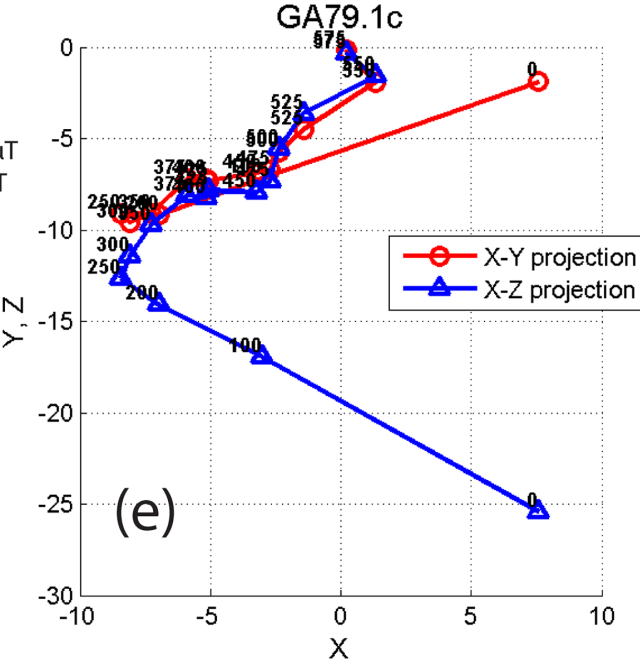

GA79.1b

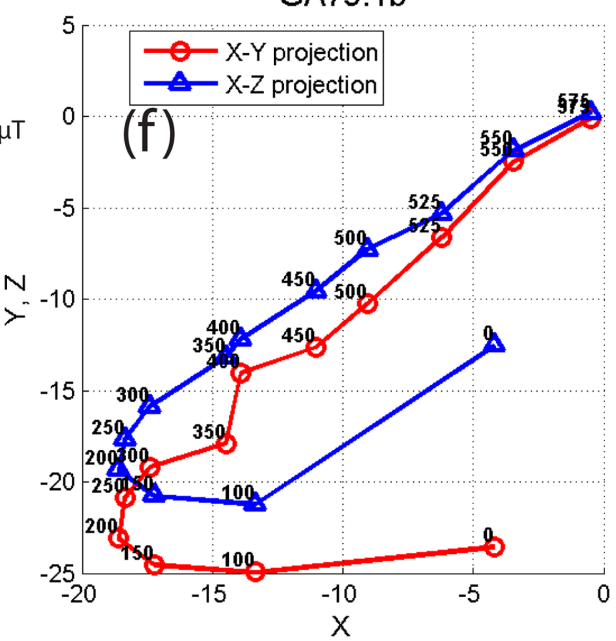

Figure 6. Arai diagrams for GA79.1c from the hybrid BZF protocol for (a) Thellier, (b) Coe*, and (c) Aitken methods (solid lines are linear regressions for fixed temperature segments of $350^{\circ}-500^{\circ} \mathrm{C}$; dashed lines are for auto-selected temperature segments), with ( $\mathrm{d}$ and e) corresponding calculated NRM thermal demagnetization vector end-point diagrams [Zijderveld, 1967] ( $\mathrm{d}$ corresponds to $\mathrm{a}$; e corresponds to both $\mathrm{b}$ and c) and actual NRM thermal demagnetization vector end-point diagram for specimen GA79.1b [Kent et al., 2010]. 
Table 2. Paleointensity Results of the Original BZF Paleointensity Experiments ${ }^{\mathrm{a}}$

\begin{tabular}{|c|c|c|c|c|c|c|c|c|c|c|c|}
\hline Sample & $\underset{\%}{\Delta \operatorname{susc}^{\mathrm{K}}}$ & $\begin{array}{c}\text { Kent10 } \\
\text { P-Int }^{\mathrm{K}}(\mu \mathrm{T})\end{array}$ & $\begin{array}{c}\text { Auto } \\
\text { Thellier } \\
\text { P-Int }(\mu \mathrm{T})\end{array}$ & $\begin{array}{c}\text { Temp Low } \\
\left({ }^{\circ} \mathrm{C}\right)\end{array}$ & $\begin{array}{l}\text { Temp High } \\
\left({ }^{\circ} \mathrm{C}\right)\end{array}$ & $\begin{array}{l}\text { Auto Coe* } \\
\text { P-Int }(\mu \mathrm{T})\end{array}$ & $\begin{array}{c}\text { Temp Low } \\
\left({ }^{\circ} \mathrm{C}\right)\end{array}$ & $\begin{array}{c}\text { Temp } \\
\text { High }\left({ }^{\circ} \mathrm{C}\right)\end{array}$ & $\begin{array}{l}\text { Auto Aitken } \\
\text { P-Int }(\mu \mathrm{T})\end{array}$ & $\begin{array}{c}\text { Temp } \\
\text { Low }\left({ }^{\circ} \mathrm{C}\right)\end{array}$ & $\begin{array}{c}\text { Temp } \\
\text { High }\left({ }^{\circ} \mathrm{C}\right)\end{array}$ \\
\hline GA78.1 & $-7.7 \%$ & 3.18 & 7.21 & 0 & 300 & 5.73 & 400 & 500 & N/A & N/A & N/A \\
\hline GA78.2 & $-16.3 \%$ & 1.70 & N/A & N/A & N/A & 1.50 & 350 & 550 & N/A & N/A & N/A \\
\hline GA78.5 & $-13.2 \%$ & 3.58 & 3.20 & 400 & 575 & 2.99 & 400 & 575 & N/A & N/A & N/A \\
\hline GA78.6 & $-5.6 \%$ & 4.74 & 4.45 & 350 & 500 & 3.44 & 400 & 500 & N/A & N/A & N/A \\
\hline GA78.8 & $-11.8 \%$ & 8.14 & 13.41 & 400 & 500 & 14.10 & 400 & 500 & 8.85 & 425 & 525 \\
\hline GA79.1 & $-26.3 \%$ & 4.10 & 3.96 & 100 & 575 & 4.78 & 100 & 500 & N/A & N/A & $\mathrm{N} / \mathrm{A}$ \\
\hline GA79.2 & $-26.3 \%$ & 3.14 & 3.80 & 0 & 575 & 3.44 & 475 & 575 & N/A & $\mathrm{N} / \mathrm{A}$ & $\mathrm{N} / \mathrm{A}$ \\
\hline GA79.3 & $-36.2 \%$ & 2.97 & 3.10 & 400 & 550 & 2.46 & 400 & 575 & 2.33 & 475 & 575 \\
\hline GA79.4 & $-30.5 \%$ & 4.98 & 7.84 & 400 & 500 & 12.23 & 100 & 500 & 4.13 & 425 & 550 \\
\hline GA79.5 & $-32.3 \%$ & 5.90 & 4.38 & 350 & 575 & 3.79 & 425 & 575 & 3.69 & 425 & 575 \\
\hline GA79.7 & $-27.9 \%$ & 8.15 & 22.65 & 0 & 500 & 6.44 & 450 & 575 & N/A & N/A & N/A \\
\hline GA79.8 & $-24.1 \%$ & 4.07 & 5.72 & 400 & 500 & 7.61 & 200 & 500 & 2.73 & 500 & 575 \\
\hline GA84.1 & $-33.3 \%$ & 4.12 & 3.14 & 450 & 550 & 2.04 & 475 & 575 & 5.34 & 100 & 550 \\
\hline GA84.2 & $-33.3 \%$ & 2.80 & 1.98 & 450 & 550 & 1.73 & 450 & 550 & 2.38 & 375 & 550 \\
\hline GA84.3 & $-29.1 \%$ & 3.67 & 3.22 & 100 & 575 & 1.87 & 475 & 575 & 3.95 & 100 & 550 \\
\hline GA84.6 & $-33.1 \%$ & 6.73 & 4.03 & 475 & 575 & 3.97 & 475 & 575 & 8.81 & 300 & 550 \\
\hline GA84.8 & $-24.0 \%$ & 3.95 & N/A & N/A & N/A & N/A & N/A & N/A & N/A & N/A & N/A \\
\hline GA85.1 & $-24.7 \%$ & 5.70 & N/A & N/A & $\mathrm{N} / \mathrm{A}$ & 5.64 & 200 & 575 & N/A & N/A & N/A \\
\hline GA85.2 & $-27.5 \%$ & 4.88 & 4.47 & 100 & 550 & 3.53 & 375 & 525 & N/A & N/A & N/A \\
\hline GA85.3 & $-22.2 \%$ & 5.30 & 3.96 & 350 & 575 & 3.92 & 350 & 575 & N/A & $\mathrm{N} / \mathrm{A}$ & N/A \\
\hline GA85.4 & $-30.6 \%$ & 4.17 & 2.83 & 400 & 575 & 3.31 & 400 & 550 & N/A & N/A & N/A \\
\hline GA85.5 & $-31.4 \%$ & 4.70 & 4.28 & 100 & 550 & 3.78 & 100 & 575 & N/A & N/A & N/A \\
\hline GA85.7 & $-40.2 \%$ & 2.63 & 1.55 & 475 & 575 & 1.98 & 375 & 575 & 2.02 & 375 & 575 \\
\hline GA85.8 & $-33.8 \%$ & 4.69 & 5.41 & 425 & 575 & 4.81 & 450 & 575 & 4.59 & 450 & 575 \\
\hline Sample $\mathrm{r}$ & number & 24 & 21 & & & 23 & & & 11 & & \\
\hline Mean & & 4.50 & 5.46 & & & 4.57 & & & 4.44 & & \\
\hline Median & & 4.14 & 4.03 & & & 3.78 & & & 3.95 & & \\
\hline Std. dev. & & 1.60 & 4.67 & & & 3.14 & & & 2.40 & & \\
\hline
\end{tabular}

${ }^{a} \Delta$ susc is the change of magnetic susceptibility from before to after heating, Kent10 ${ }_{350-575}$ P-Int is the paleointensity estimated using only two points at $350^{\circ} \mathrm{C}$ and $575^{\circ} \mathrm{C}$, Auto (Thellier, Coe* and Aitken) P-Int are paleointensities estimated using automatic temperature range selection for these three protocols, Temp Low and Temp High mark the automatic selected temperature ranges for each protocol. Columns superscripted by "K" are data from Kent et al. [2010].

dominated by MD particles, the outcome may be different due to the low- and high-temperature pTRM tails associated with MD behavior.

[19] After plotting the vector end-point [Zijderveld, 1967] and Arai diagrams [Nagata et al., 1963] (Figure 6) for the two possible NRM outcomes and the three classic paleointensity methods from the BZF protocol, we used a relatively generous set of criteria to automatically calculate the paleointensities using the program ThellierTool v.4.22 [Leonhardt et al., 2004]: number of points $(\mathrm{N}) \geq 4$; standard deviation $(\mathrm{Std}) \leq 0.2$; fraction of $\mathrm{NRM}(\mathrm{f}) \geq 0.3$; quality factor $(\mathrm{q})>0$; maximum angular deviation $(\mathrm{MAD}) \leq 20^{\circ}$; alpha $\leq 20$; relative check error $(\mathrm{dCK}) \leq 10$; cumulative check diff $(\mathrm{dPAL}) \leq 15$; normalized tail of pTRM $\left(\mathrm{dt}^{*}\right) \leq 8$; relative intensity $\operatorname{diff} \leq 25$; relative $\mathrm{AC}$ error $(\mathrm{dAC}) \leq 15$. We set the program to use as many data points as possible to estimate the paleointensity value. The paleointensity results and automated criteria temperature segments are listed in Table 2.

[20] As illustrated in Figure 6 for a typical specimen (GA79.1c), using the above set of criteria to automatically calculate paleointensity resulted in a variety of selected temperature segments and paleointensity outcomes for the three standard methods. The NRM thermal demagnetization vector end-point diagram in Figure $6 \mathrm{~d}$ is calculated from $\mathrm{B}$ and $\mathrm{F}$ steps in the BZF experiment, corresponding to the Arai diagram in Figure 6a. Figure 6e is based on $\mathrm{Z}$ steps, corresponding to Arai diagrams in both Figures $6 \mathrm{~b}$ and c. Figure $6 \mathrm{f}$ is the actual NRM thermal demagnetization vector endpoint diagram for specimen GA79.1b [Kent et al., 2010]. Although strong VRM components can be identified up to $250^{\circ} \mathrm{C}$, sample GA79.1 shows a dominant primary TRM component going toward the origin as temperature increased to the Curie temperature. Use of a fixed middle temperature segment $\left(350^{\circ}-500^{\circ} \mathrm{C}\right)$, which may be a relatively small fraction of the NRM yet avoids lowtemperature VRM and high-temperature alteration, yielded much more consistent values so all three standard methods yield almost the same value for site GA-X, with median paleointensity estimates of 5.63, 5.67, and 5.55 $\mu \mathrm{T}$, for the Thellier, Coe*, and Aitken protocols, respectively (Figure 7 and Table 2). By comparison, the $350^{\circ}-575^{\circ} \mathrm{C}$ twopoint paleointensity estimates for $24 \mathrm{~b}$ specimens 

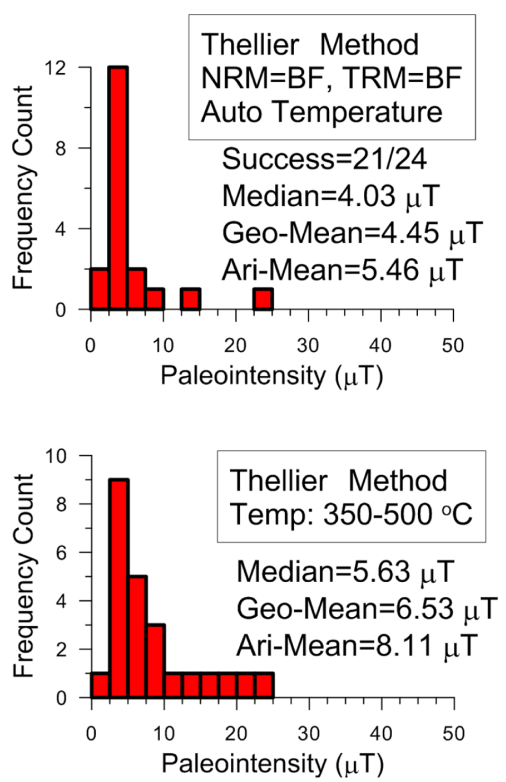
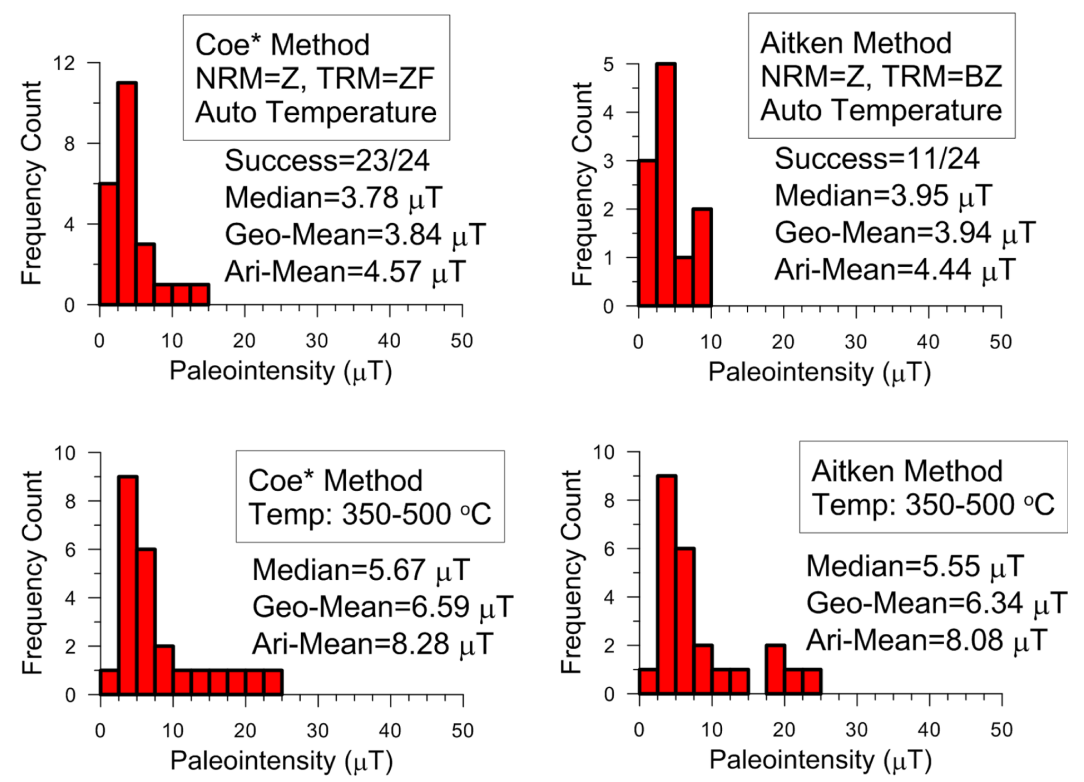

Figure 7. Histograms of BZF paleointensity results for different calculation methods for (top) automated temperature selection and (bottom) fixed $350^{\circ}-500^{\circ} \mathrm{C}$ temperature range.

from site GA-X [Kent et al., 2010] yielded a median value of $4.14 \mu \mathrm{T}$ (Table 2). The relatively more scattered outcomes of the automated-selection compared to the fixed temperature segment paleointensity calculation is clearly seen in the histograms for the 24 results (Figure 7). However, the risk of using a fixed temperature segment for all the samples is that the overall site-mean value could be biased due to concave-up Arai diagrams for MD grain contribution.

\subsection{Correction for MD Concave-Up Arai Diagrams by Repeating BZF experiments}

[21] In order to perform a correction for the MD concave-up pattern on the Arai diagram, we gave each specimen a total TRM (tTRM) by cooling from $575^{\circ} \mathrm{C}$ in the presence of a laboratoryapplied field $(15 \mu \mathrm{T})$ along the $\mathrm{X}$ axis, i.e., perpendicular to the applied-field direction for the initial BZF experiments. The BZF protocol described above was then repeated with the laboratoryapplied tTRM as a synthetic NRM, using the same laboratory-applied field (both the same direction and intensity) and target temperatures as before. We name the Arai diagrams for this repeated BZF experiment "Arai signatures" that represent only the TRM recording properties of the specimens, if no severe thermal alteration occurs in the laboratory heating process. Due to the range of effective magnetic grain sizes in the specimens, which cause differences in the resulting Thellier series protocols, each specimen is expected to have a unique Arai signature associated with a particular experiment protocol (Thellier, Coe*, and Aitken).

[22] We performed MD corrections by plotting the NRM unblocking remaining from the first BZF against the laboratory-applied tTRM unblocking from the repeated BZF to generate the corrected Arai diagrams. The corrected Arai diagrams, therefore, use the Arai signatures to neutralize MD concave-up contributions in the original Arai diagrams by plotting original NRM unblocking versus synthetic NRM unblocking, and should thus provide unbiased paleointensity estimates.

[23] All specimens from site GA-X produce concave-up Arai diagrams in both the first and second BZF experiments, which confirms that all specimens contain portions of MD magnetization carriers as anticipated by the hysteresis results. Representative results produced from the first and second BZF experiments for a specimen are shown in Figure 8a (also see Figures 9 and 10 for more concave-up Arai diagrams). The MD contribution $(\delta \mathrm{MD})$ can be parameterized from the ratio of the area enclosed between the Arai signature and the line joining the beginning and end points (green dashed line in Figure 8a) divided by the triangular area enclosed by this line and the axes. For example, $\delta \mathrm{MD}$ for the specimen GA79.8c in Figure 8a is 0.183 . However, because the line connecting the 

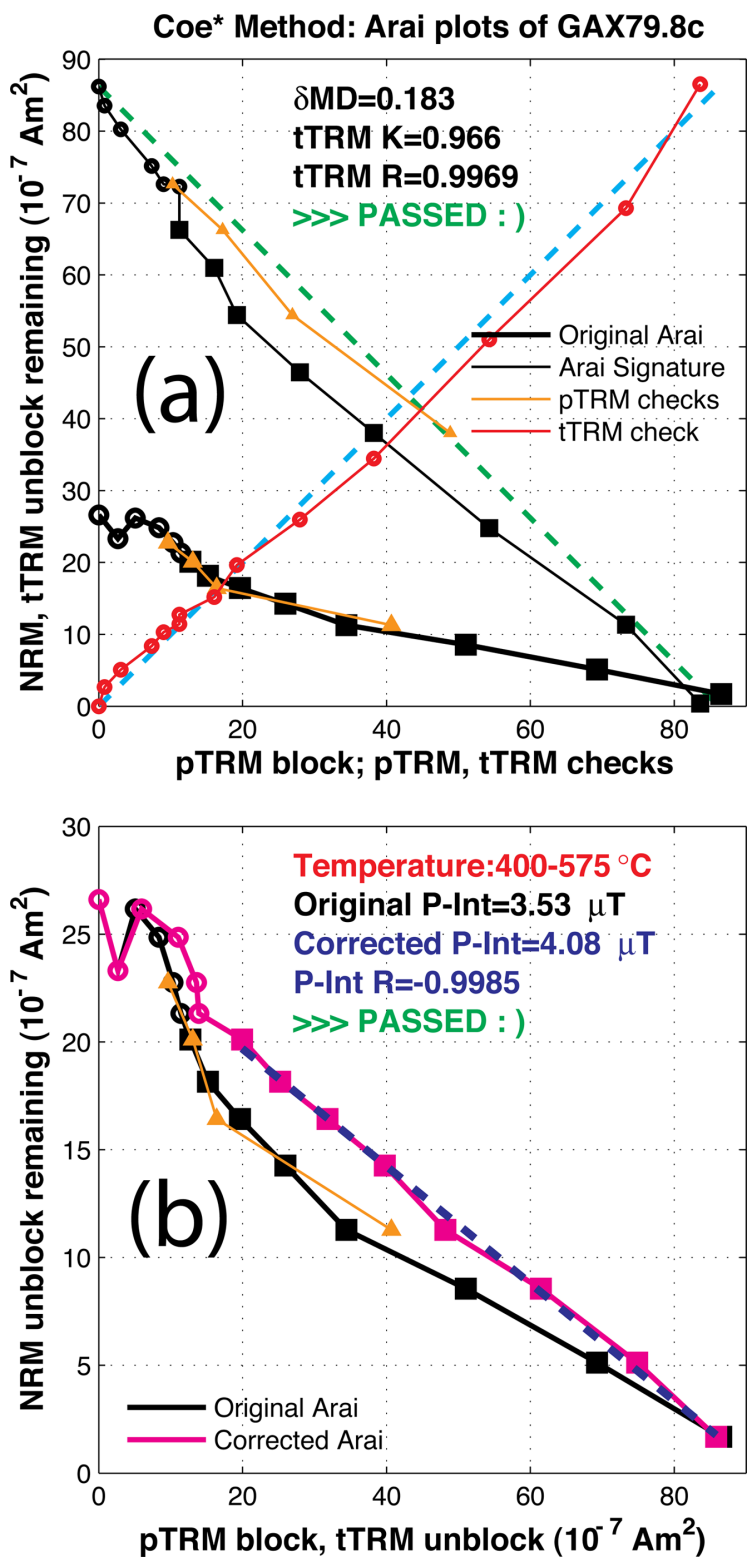

Figure 8. Paleointensity results for GA79.8c from the first and repeated BZF experiments, calculated using the Coe* method according to the highest quality control factors. (a) Arai diagram of the first (thick black line) and repeated (thin black line) BZF experiments, with circles indicating temperatures $20^{\circ}-375^{\circ} \mathrm{C}$ and squares indicating $400^{\circ}-575^{\circ} \mathrm{C}$. The orange line connects the pTRM checks. The red line represents the first BZF pTRM gains versus the pTRM gains in the repeated BZF experiment (tTRM check). The light blue dashed line is the 1:1 ratio of the first and the repeated pTRM gains. The green dashed line is the theoretical linear prediction of Arai diagrams for SSD grains for repeated BZF experiment. (b) Arai diagrams of the first (thick black line) BZF experiments with pTRM checks (orange line). The pink line is the original NRM unblocking remaining from the first BZF experiment versus the laboratory-applied tTRM unblocking from the repeated BZF experiment with the blue dashed line representing the linear regression for the $400^{\circ}-575^{\circ} \mathrm{C}$ temperature segment.
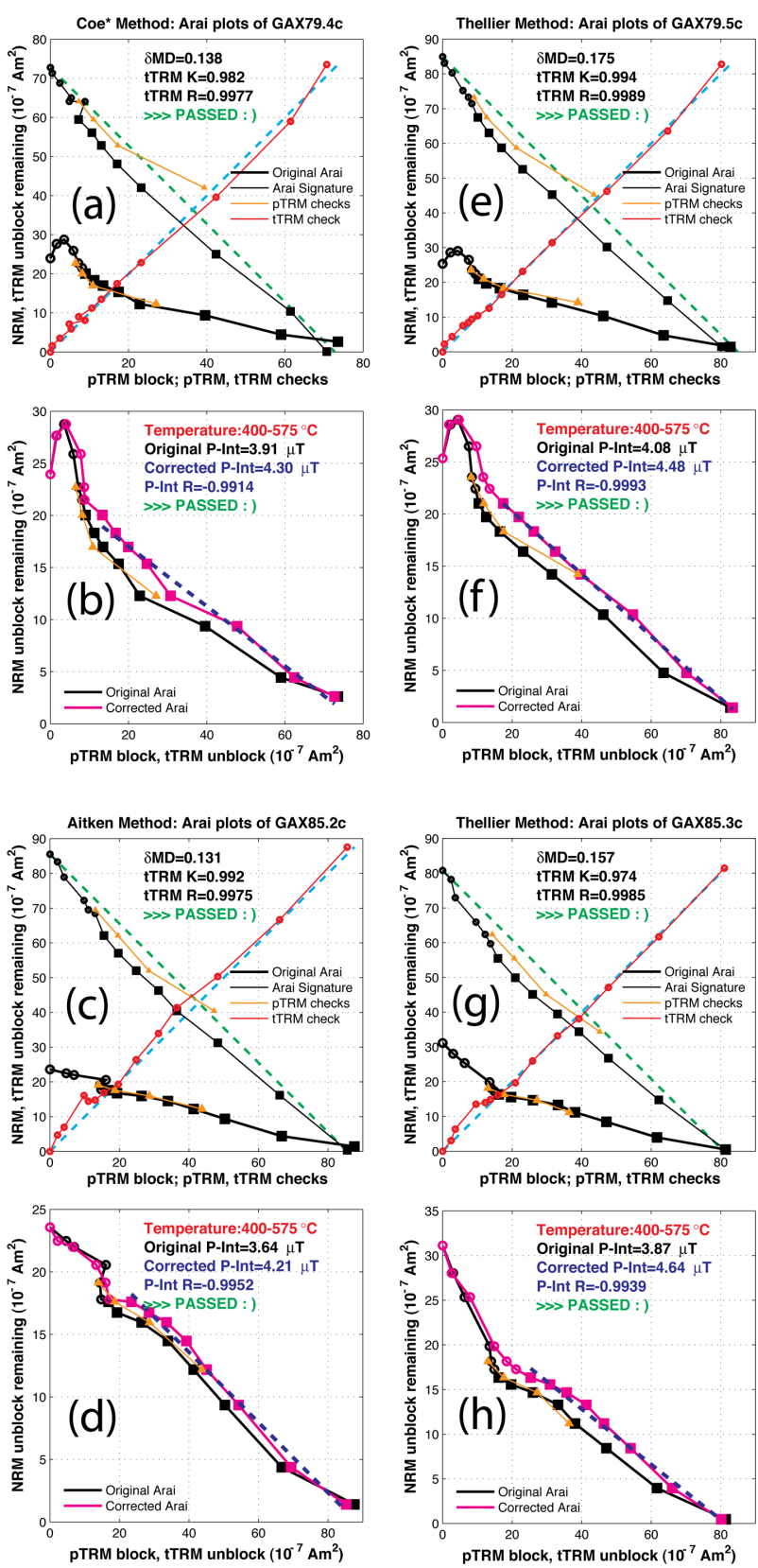

Figure 9. Paleointensity results for four of the nominally qualified specimens (GA79.4c, GA79.5c, GA85.2c, and GA85.3c).

beginning and end points in the Arai diagram is not affected by the $\delta \mathrm{MD}$ content, the total TRM (tTRM) should be reproducible as shown in previous work [Levi, 1977; Xu and Dunlop, 2004]. A plot of the first against repeated pTRM acquisitions can be used as an indicator of thermophysicochemical alterations (red line in Figure 8a), whose linearity decreases and slope diverts from 1 if the recording capability of pTRM changes from the first to the second BZF experiment. We 

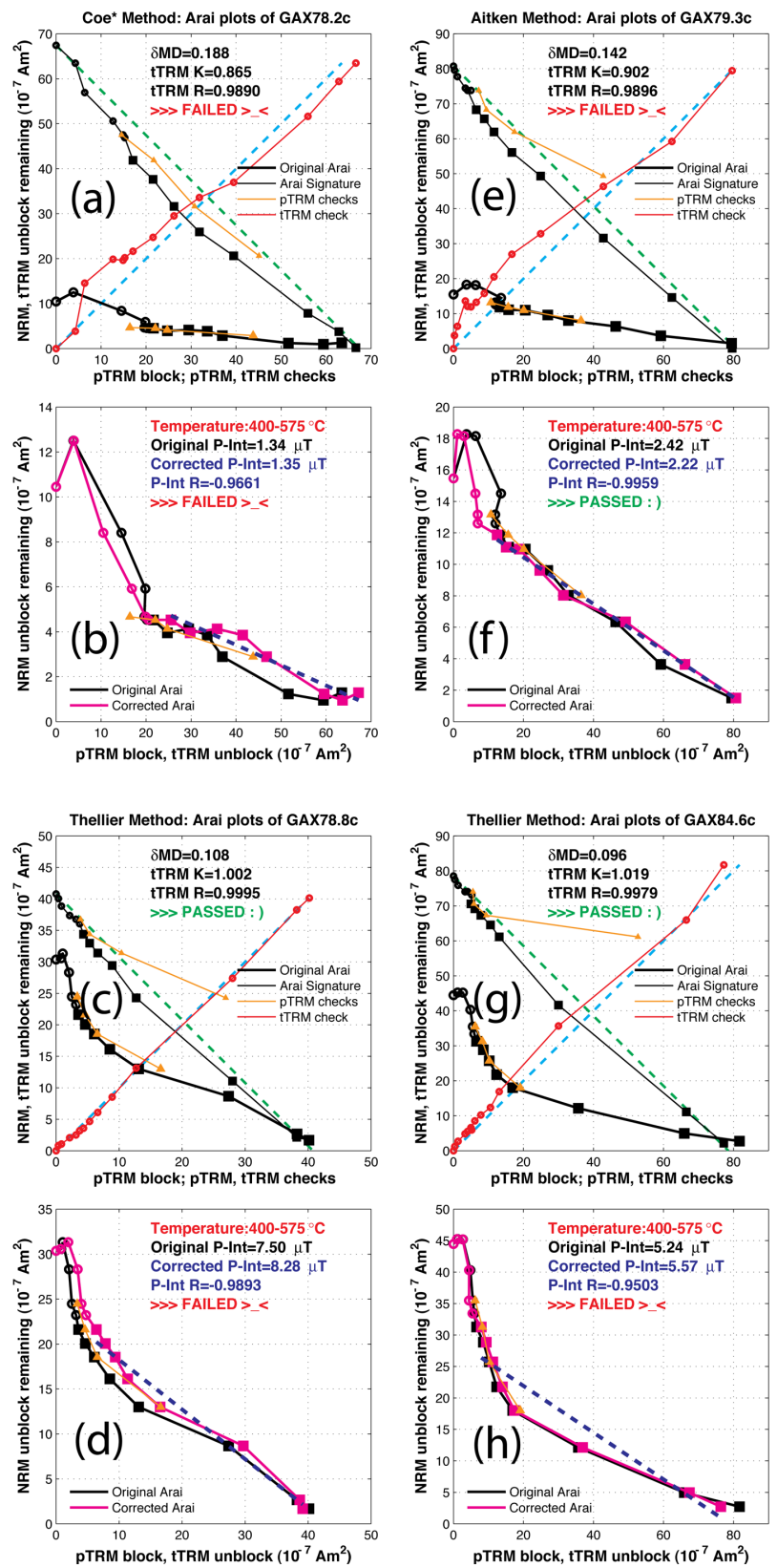

Figure 10. Paleointensity results for four of the disqualified specimens (GA78.2c, GA78.8c, GA79.3c, and GA84.6c).

quantify this expected agreement by using its least-squares fit slope (tTRM-K) and linear regression correlation coefficient (tTRM-R). We call it "tTRM check" for alteration, as opposed to "pTRM checks" as shown in yellow lines for both the original Arai diagram and Arai signature. The pTRM that is carried by a tTRM check is equivalent to type TRRM $^{*}$ defined by Shcherbakov et al. [1993]. The tTRM check offers a quantitative measurement of specimen alteration before and after it has been thoroughly heated to its Curie tem- perature, as opposed to the stepwise pTRM checks.

[24] The NRMs in both the first and second BZF experiments obviously have contributions from secondary VRMs, as evidenced by the component structure in vector end-point demagnetization diagrams (Figure 6), which affect the first BZF experiments and cause curvatures of the corrected Arai diagrams in the low temperature ranges up to $350^{\circ}-400^{\circ} \mathrm{C}$. Accordingly, we used the corrected Arai diagram from $400^{\circ} \mathrm{C}$ to $575^{\circ} \mathrm{C}$ to estimate the unbiased paleointensity for a specimen and the associated absolute value of linear regression correlation coefficient (P-Int-R) as a representative measure of the qualities of that estimate.

[25] Specimen GA79.8c gives tTRM-K $=0.9663$, $\mathrm{tTRM}-\mathrm{R}=0.9969$ (Figure 8a), and P-Int$\mathrm{R}=0.9985$ (Figure $8 \mathrm{~b}$ ). With the support of good linearities of both the tTRM check and the corrected Arai diagram, specimen GA79.8c provides a reliable paleointensity estimation of $4.08 \mu \mathrm{T}$ (Figure $8 b$ ). In order to systematically assess the quality of paleointensity estimates, we arbitrarily set a simple quality parameter threshold as follows: the absolute values of tTRM-R and the PInt-R need to be greater than 0.9900 . We used the calculation method (Thellier, Coe*, and Aitken) that provided the best P-Int-R and then tTRM-R to estimate the paleointensity (e.g., we used the Coe* method for specimen GA79.8c).

[26] Typical results calculated by the best methods for four representative specimens that meet the aforestated quality criteria (GA79.4c-Coe*, GA7 9.5c-Thellier, GA85.2c-Aitken, and GA85.3cThellier) are shown in Figure 9. Together, they provide well-clustered paleointensity estimates that range from 4.21 to $4.64 \mu \mathrm{T}$. Beside the five specimens shown in Figures 8 and 9, six other specimens from site GA-X also pass the 0.9900 qualification criteria, making the total success rate 11 out of 24 (Table 3 ).

[27] Typical results calculated by the best method for each of four representative specimens that fail the earlier described quality criteria are presented in Figure 10. Specimen GA78.2c failed both tTRM-R and P-Int-R; GA79.3c failed tTRM-R but passed P-Int-R; GA78.8c and GA84.6 both passed tTRM-R but failed P-Int-R. For specimens GA78.2 and GA79.3, the tTRM checks reveal large non-linear features, which indicate thermophysicochemical alterations between the same temperature steps of the first and second BZF experiments. It is therefore reasonable to conclude 
Table 3. Paleointensity Results After Using Arai Signatures to Conduct MD Corrections ${ }^{\mathrm{a}}$

\begin{tabular}{|c|c|c|c|c|c|c|c|c|c|c|c|}
\hline Specimen & $\begin{array}{c}\text { Rock } \\
\text { Magnetic } \\
\text { Pre-selection }\end{array}$ & $\begin{array}{l}\text { Quality } \\
\text { Control }\end{array}$ & $\begin{array}{c}\text { Best } \\
\text { Method }\end{array}$ & $\begin{array}{c}\text { Original } \\
\text { P-Int }(\mu \mathrm{T})\end{array}$ & $\begin{array}{c}95 \% \\
\text { Confident } \\
\text { Range }(\mu \mathrm{T})\end{array}$ & $\begin{array}{l}\text { Corrected } \\
\text { P-Int }(\mu \mathrm{T})\end{array}$ & $\begin{array}{c}95 \% \\
\text { Confident } \\
\text { Range }(\mu \mathrm{T})\end{array}$ & $\delta \mathrm{MD}$ & $\begin{array}{l}\text { tTRM-K } \\
\text { Regression } \\
\text { Slope }\end{array}$ & $\begin{array}{c}\text { tTRM-R } \\
\text { Correlation } \\
\text { Coefficient }\end{array}$ & $\begin{array}{c}\text { Corrected } \\
\text { P-Int-R } \\
\text { Correlation } \\
\text { Coefficient }\end{array}$ \\
\hline GA78.1c & Yes & Failed & Coe* & 3.52 & 0.46 & 3.55 & 0.38 & 0.22 & 0.9910 & 0.9980 & -0.9883 \\
\hline GA78.2c & No & Failed & Coe* & 1.34 & 0.26 & 1.35 & 0.25 & 0.19 & 0.8646 & 0.9890 & -0.9661 \\
\hline GA78.5c & No & Passed & Coe* & 2.93 & 0.43 & 3.08 & 0.30 & 0.16 & 0.9289 & 0.9970 & -0.9907 \\
\hline GA78.6c & Yes & Passed & Thellier & 2.99 & 0.49 & 3.38 & 0.24 & 0.11 & 0.9047 & 0.9963 & -0.9948 \\
\hline GA78.8c & Yes & Failed & Thellier & 7.50 & 0.83 & 8.28 & 0.86 & 0.11 & 1.0024 & 0.9995 & -0.9893 \\
\hline GA79.1c & No & Failed & Thellier & 2.88 & 0.40 & 3.69 & 0.77 & 0.28 & 0.9094 & 0.9881 & -0.9588 \\
\hline GA79.2c & Yes & Passed & Coe* & 3.43 & 0.24 & 3.78 & 0.19 & 0.20 & 0.9992 & 0.9975 & -0.9975 \\
\hline GA79.3c & No & Failed & Aitken & 2.42 & 0.19 & 2.22 & 0.14 & 0.14 & 0.9022 & 0.9896 & -0.9959 \\
\hline GA79.4c & No & Passed & Thellier & 4.08 & 0.50 & 4.47 & 0.37 & 0.15 & 0.9894 & 0.9981 & -0.9932 \\
\hline GA79.5c & Yes & Passed & Thellier & 4.08 & 0.25 & 4.48 & 0.12 & 0.18 & 0.9941 & 0.9989 & -0.9993 \\
\hline GA79.7c & Yes & Passed & Coe* & 7.05 & 0.96 & 7.64 & 0.63 & 0.20 & 1.0324 & 0.9988 & -0.9932 \\
\hline GA79.8c & Yes & Passed & Coe* & 3.53 & 0.44 & 4.08 & 0.16 & 0.18 & 0.9663 & 0.9969 & -0.9985 \\
\hline GA84.1c & No & Failed & Thellier & 2.79 & 0.79 & 2.85 & 0.59 & 0.15 & 0.9916 & 0.9944 & -0.9587 \\
\hline GA84.2c & No & Failed & Aitken & 1.57 & 0.44 & 1.52 & 0.32 & 0.15 & 0.9539 & 0.9918 & -0.9574 \\
\hline GA84.3c & No & Failed & Aitken & 2.34 & 0.64 & 2.45 & 0.53 & 0.17 & 1.0331 & 0.9950 & -0.9556 \\
\hline GA84.6c & No & Failed & Thellier & 5.24 & 1.29 & 5.57 & 1.28 & 0.10 & 1.0190 & 0.9979 & -0.9503 \\
\hline GA84.8c & No & Failed & Aitken & 2.74 & 0.59 & 2.40 & 0.51 & 0.17 & 0.9315 & 0.9847 & -0.9570 \\
\hline GA85.1c & No & Failed & Aitken & 5.46 & 0.81 & 5.73 & 0.90 & 0.16 & 0.9361 & 0.9937 & -0.9760 \\
\hline GA85.2c & Yes & Passed & Aitken & 3.64 & 0.31 & 4.21 & 0.29 & 0.13 & 0.9925 & 0.9975 & -0.9952 \\
\hline GA85.3c & Yes & Passed & Thellier & 3.87 & 0.30 & 4.64 & 0.36 & 0.16 & 0.9736 & 0.9985 & -0.9939 \\
\hline GA85.4c & No & Passed & Aitken & 2.66 & 0.36 & 2.65 & 0.26 & 0.11 & 0.9349 & 0.9900 & -0.9906 \\
\hline GA85.5c & Yes & Passed & Coe* & 3.77 & 0.34 & 4.16 & 0.30 & 0.13 & 0.9864 & 0.9983 & -0.9947 \\
\hline GA85.7c & Yes & Failed & Thellier & 1.98 & 0.39 & 1.81 & 0.35 & 0.12 & 0.8640 & 0.9878 & -0.9635 \\
\hline GA85.8c & No & Failed & Aitken & 5.98 & 1.65 & 7.26 & 1.57 & 0.06 & 0.9585 & 0.9966 & -0.9556 \\
\hline $\begin{array}{l}\text { Qualified } \\
\text { Specime } \\
\text { Number }\end{array}$ & & 11 & & 11 & 11 & 11 & 11 & 11 & 11 & 11 & 11 \\
\hline Mean & & & & 3.82 & 0.42 & 4.23 & 0.29 & 0.16 & 0.9729 & 0.9971 & -0.9947 \\
\hline Median & & & & 3.64 & 0.36 & 4.16 & 0.29 & 0.16 & 0.9864 & 0.9975 & -0.9947 \\
\hline Std. dev. & & & & 1.17 & 0.20 & 1.29 & 0.14 & 0.03 & 0.0369 & 0.0025 & 0.0029 \\
\hline
\end{tabular}

${ }^{\mathrm{a}}$ Original P-Int is the paleointensity estimated from the original BZF experiment, Corrected P-Int is the MD Arai signature corrected paleointensity estimated using both original and repeated BZF experiments, $\delta \mathrm{MD}$ is the MD contribution parameter, tTRM-K and tTRM-R are the linear regression slope and correlation coefficient of tTRM check, Corrected P-Int-R correlation coefficient is the linear regression correlation coefficient of corrected Arai diagram. Bold raws are qualified paleointensity results and their statistics.

that they are disqualified to provide reliable paleointensity estimates. Paleointensity values from these specimens $(1.35 \mu \mathrm{T}$ and $2.22 \mu \mathrm{T})$ are also consistently lower than the values estimated from the qualified specimens. For specimens GA78.8c and GA84.6c, tTRM checks pass, which indicates no alteration. However, their corrected Arai diagrams fail to present linear features as expected. Paleointensity values from these specimens $(8.28$ and $5.57 \mu \mathrm{T}$ ) are consistently high compared to the values estimated from the qualified specimens. The reason why they fail to yield linear corrected Arai diagrams is complicated, which we attempt to explain below. All 24 of the results from the new MD paleointensity technique for site GA-X are listed in Table 3.

[28] Vector end-point diagrams for thermal demagnetization of NRM of selected "b" specimens from Kent et al. [2010] are shown in Figure 11. Despite clearly identified low-temperature VRM components up to $300^{\circ}-400^{\circ} \mathrm{C}$, most of the samples from site GA-X yield trajectories going toward the origin up to the Curie temperature, consistent with primary TRMs. The "c" counterparts of specimens GA79.8b, GA79.5b, and GA85.3b (Figures 11a-c) provide acceptable corrected paleointensity results (Figures 8 and 9), whereas the "c" specimens of GA78.8, GA79.3, and GA84.6 (Figures 11d-f) provide failed paleointensity results (Figure 10).

\section{5. pTRM Checks}

[29] The pTRM check is usually taken for granted as a valid indicator of thermophysicochemical alterations. However, for GA79.5c (Figures 9e and f), and GA79.8c (Figures 8a and b), the pTRM checks are not consistent with the original pTRM acquisition for both original Arai diagrams and Arai signatures, yet data for these samples yield two of the best P-Int-Rs, and provide very satisfactory paleointensity estimates. This suggests that the pTRM checks are false alarms in these cases 

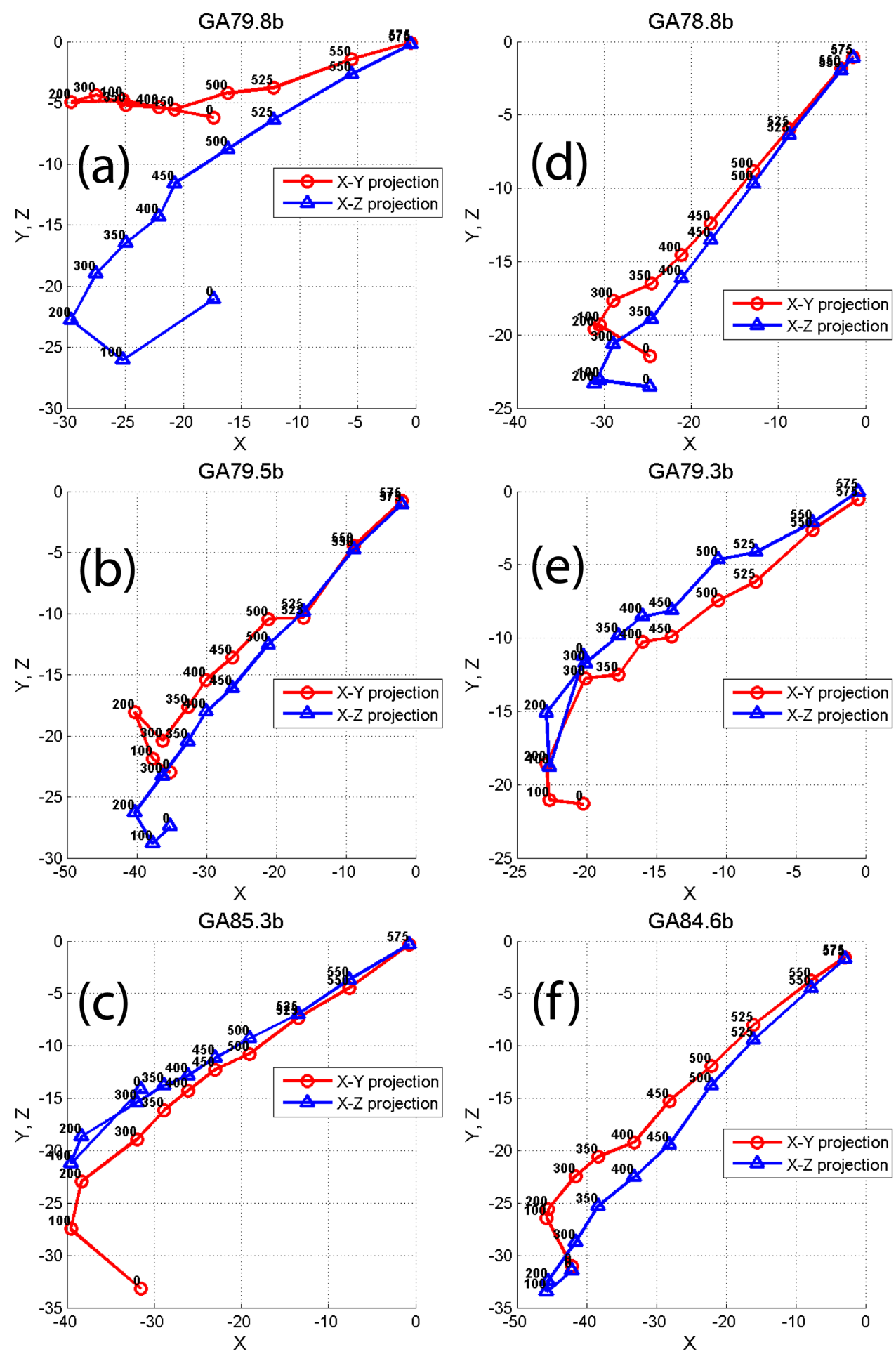

Figure 11. NRM thermal demagnetization vector end-point diagrams [Zijderveld, 1967] for " $b$ " specimens (qualified paleointensity results: (a) GA79.8b, (b) GA79.5b, and (c) GA85.3b; disqualified paleointensity results: (d) GA78.8b, (e) GA79.3b, and (f) GA84.6b) from site GA-X from Kent et al. [2010].

and more generally suggests that it may be inappropriate to automatically disqualify paleointensity results on the basis of pTRM checks. Nevertheless, the tTRM checks (tTRM-K and
tTRM-R) that we developed in this study, which compare the stepwise pTRM acquisitions of the first BZF against the second BZF experiments, behaved well. The difference between the pTRM 
check and tTRM check is that the tTRM check compares exactly the same processes (pTRM stepwise blocking for the exact same temperature step), whereas the pTRM check compares somewhat different processes (the original pTRM blocking and the back-check pTRM blocking after a zero-field step partial thermal demagnetization). For SSD specimens, the pTRM checks may work as intended. But for MD specimens, the partial demagnetization step between the original pTRM acquisition and pTRM check acquisition is not completely clean due to the non-linear (concaveup) Arai diagram. Thus, the signals in pTRM checks reflect not only the thermophysicochemical alteration but also the non-ideal behavior of MD contributions. We therefore suggest that the tTRM check is a more powerful and appropriate technique to identify alterations instead of the pTRM check for samples with significant MD contributions.

[30] Based on many previous works that have experimentally and theoretically studied effects of thermochemical alteration over the blocking spectra of SSD particles [McClelland, 1996; Draeger et al., 2006; Yamamoto, 2006; Fabian, 2009], we also suggest that the pTRM check should not be automatically relied on for SSD samples. Specimens GA78.8c (Figure 10c) and GA84.6c (Figure $10 \mathrm{~g}$ ) have satisfactory pTRM checks for the original Arai diagrams as well as acceptable tTRM checks, which indicate little alteration from room temperature to the Curie temperature. However, their corrected Arai diagrams are still curved at high temperatures $\left(475^{\circ}-500^{\circ} \mathrm{C}\right)$, which is not likely to be caused by sudden demagnetization of VRM. This is because both the pTRM check and the tTRM check are only capable of detecting alteration of ferrimagnetic grains with blocking/ unblocking temperatures lower than the current checking temperature. If a specimen is heated from step $T_{i}$ to step $T_{i+1}$, particles with blocking/ unblocking temperatures between $T_{i}$ and $T_{i+1}$ may have altered but are not able to be detected by pTRM check back to $T_{i}$. In the BZF experiments, it happens during the first back-field heating steps. But the pTRM or tTRM checks back to $T_{i}$ are not capable of detecting such alteration because they occur outside of the blocking/unblocking temperature ranges for those grains. Figure 12 illustrates the actual data from GA84.6 by using the Coe paleointensity protocol. For the temperature step at $550^{\circ} \mathrm{C}$, the sample is heated in zero-field for step 1; and then in-field for step 2; followed by another in-field heating to $500^{\circ} \mathrm{C}$ for step 3 to per-

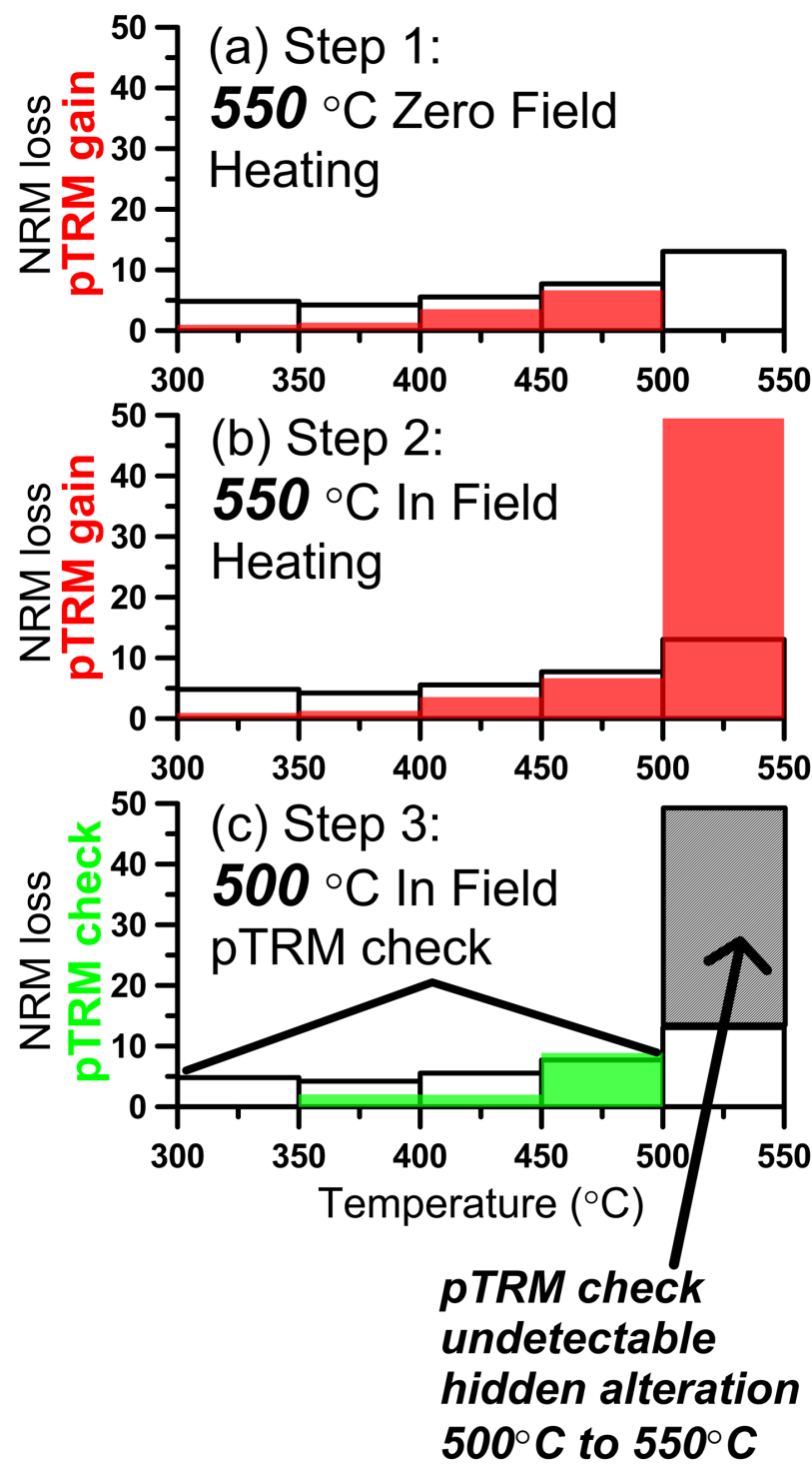

Figure 12. pTRM model from actual GA84.6 data, which indicates the hidden pTRM increase that cannot be detected by pTRM checks. White boxes are NRM spectra calculated from GA84.6b thermal demagnetization experiment from Kent et al. [2010]. Red bars are pTRM acquisitions for each temperature segment calculated using $\mathrm{Coe}^{*}$ method from GA84.6c. Green bars are pTRM checks for each temperature segment method from GA84.6c up to $500^{\circ} \mathrm{C}$. Gray bar is the hidden alteration from $500^{\circ}-550^{\circ} \mathrm{C}$ (happened during (a) Step 1; becames visible after (b) Step 2) that cannot be detected by pTRM check in (c) Step 3.

form pTRM check (Figure 12). But the sudden TRM recording capability increase for the grains that have blocking/unblocking temperatures between $500^{\circ} \mathrm{C}$ and $550^{\circ} \mathrm{C}$ (gray bar in Figure 12) cannot be detected by a pTRM check back to $500^{\circ} \mathrm{C}$, which only applies to those grains that have blocking/unblocking temperatures between 


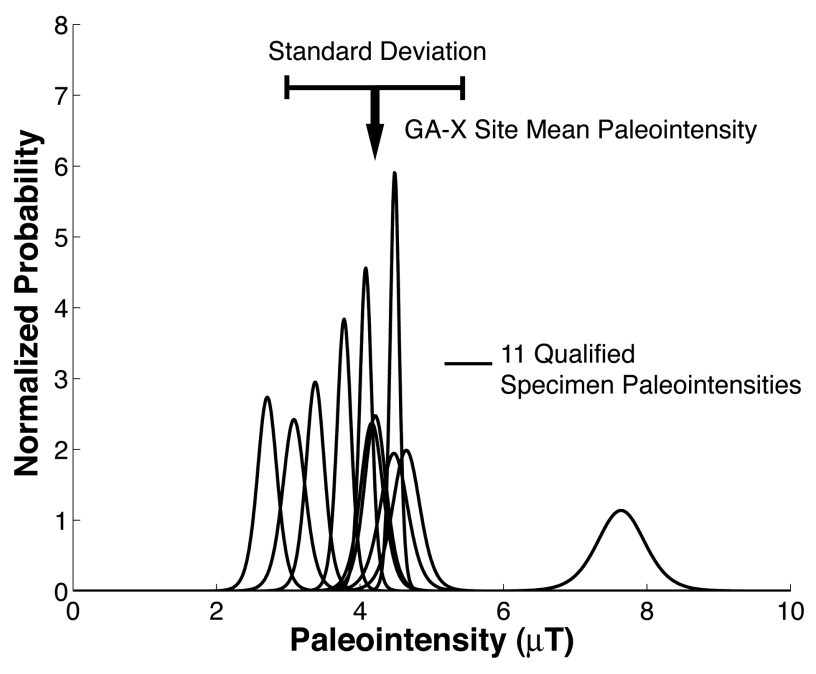

Figure 13. Paleointensity probability distributions for 11 qualified results from site GA-X.

room temperature and $500^{\circ} \mathrm{C}$. The alternative way to check this type of hidden ( $T_{i}$ to $\left.T_{i+1}\right)$ alteration is to see if the corrected Arai diagram is linear. If the corrected Arai diagram is linear from a certain high temperature that avoids VRM $\left(400^{\circ} \mathrm{C}\right.$ selected here) to the maximum blocking temperature near the Curie point $\left(575^{\circ} \mathrm{C}\right.$ in the $\mathrm{BZF}$ experiments), this would likely indicate not only that the corrected Arai diagram provides a reliable paleointensity estimate but also that the specimen has not experienced serious thermophysicochemical alteration. Fortunately for site GA-X, 11 of 24 specimens behaved in this way (Figures 8, 9, and 13 and Table 3).

[31] We find that pTRM checks from repeated BZF experiments work well for detecting thermophysicochemical alterations that occurred during the first back-field heating steps of the original BZF experiment (Figures 10c, e, and g), with the hidden increased pTRM carrying capabilities coinciding with the pTRM check failures over $500^{\circ} \mathrm{C}$. This is because when the hidden pTRM recording capability increases due to alteration, its high and low temperature tails also increase, allowing them to be detectable during repeated BZF experiments with the pTRM checks.

[32] De Groot et al. [2011] reported that small magnetite grains $(<\sim 3 \mu \mathrm{m})$ in a lava flow sample appeared to undergo greater change in magnetic domain configurations during heating than larger grains $(>\sim 10-15 \mu \mathrm{m})$. This is consistent with our results. Hysteresis loops (Figure 3) and Day plots (Figure 4) provide evidence that samples with larger MD contributions (GA79.5) tend to undergo less thermophysicochemical alteration, whereas samples more dominated by a SSD contribution (GA84.6) tend to undergo greater thermophysicochemical alteration. Moreover, the before and after heating rock magnetic results (stable $\mathrm{J}_{\mathrm{s}}$-T curves but varying hysteresis properties) also reveal that the thermophysicochemical alteration path is from more MD toward more SSD properties. The finer-grain size the original grains are (i.e., greater the $\mathrm{Mr} / \mathrm{Ms}$ for the assemblage), the more likely they tend to undergo this kind of thermophysicochemical alteration in domain status, so SSD-like samples undergo greater alteration than MD-behaved samples. Thermophysicochemical alteration in the SSDlike samples are often of the hidden $\left(T_{i}\right.$ to $\left.T_{i+1}\right)$ type (Figure 10: GA78.8c and GA84.6c), which is not detected by pTRM or even tTRM checks. The benefit of using samples containing MD grains is that, even if some thermophysicochemical alteration occurs, the MD grains remain MD grains. Arai diagrams predicted by MD field blocking theory $[X u$ and Dunlop, 2004] for MD grains tend to vary little even with large MD grain size changes. The Arai signature of a sample containing MD grains will therefore remain similar to its original Arai diagram, which still closely represents the original TRM recording properties of the sample.

\section{Discussion}

[33] Certain sets of data qualifying criteria similar to those used in this study for automatic estimations have usually been used to include or exclude specimens in most previous paleointensity studies. The main judging factors are usually the linearity of the Arai diagram and the pTRM checks. This often results in variable temperature segments chosen by the criteria to estimate paleointensities. Automatic criteria-based paleointensity selection for the GA-X samples (Table 2) yields temperature segments as low as room temperature and as high as the Curie temperature for paleointensity estimates. This is obviously inappropriate because the low and high temperature ranges of Arai diagram are likely to be affected by VRM and thermophysicochemical alteration, respectively. Moreover, for non-SSD specimens with concave-up Arai diagrams, selection of inconsistent temperature segments introduces large random errors, which usually overwhelm and disguise the potential biases. We therefore used a fixed temperature segment of $400^{\circ}-575^{\circ} \mathrm{C}$ for the corrected paleointensity estimations, in order to avoid low temperature VRM and to minimize uncertainties from using automatically selected temperatures. 
[34] Based on the rock magnetic data and results of our repeated BZF experiments, we developed a simple set of sample pre-selection criteria in an attempt to increase the success rate of future paleointensity estimations. If we only used samples with $\mathrm{M}_{\mathrm{r}} / \mathrm{M}_{\mathrm{s}}$ less than 0.19 (to avoid SSD thermal alteration) and $\mathrm{B}_{\mathrm{cr}}$ larger than $20 \mathrm{mT}$ (to avoid unstable remanences) (see Tables 1 and 3), our success rate would have increased from $46 \%(11 / 24)$ to $64 \%$ (7/ 11). However, this would also reduce the qualified paleointensity estimates from 11 to 7 .

[35] The Arai signature correction technique developed in this study offers an unbiased approach to estimate reliable paleointensities from MD specimens. The traditional three Thellier laws (additivity, reciprocity, and independence of pTRM [Thellier, 1938]) do not have to be completely met to provide accurate paleointensity estimations. However, this MD technique still requires the samples to be relatively thermophysicochemically stable, which happens to be largely true for samples from site GA-X.

[36] Sbarbori et al. [2009] used the IZZI method to estimate paleointensities from volcanic rocks from Isla Socorro, Mexico. They repeated their original IZZI method after giving their samples laboratory-applied tTRM cooling from $610^{\circ} \mathrm{C}$ at an orthogonal direction to the laboratory field used in the IZZI experiments, in an attempt to effectively perform the "Arai signature correction" that we propose in this study. However, for the repeated IZZI experiment, their non-ideal Type-II specimens, which yielded typical concave-up original Arai diagrams [Sbarbori et al., 2009, Figure $8 \mathrm{~b}$ ], produced almost linear Arai signatures [Sbarbori et al., 2009, Figure 10b]. Thus they did not manage to perform the MD correction and gave up the idea of repeating the original paleointensity experiments. In this study, by studying igneous rock samples of various overall grain size (domain status), we conclude that the Type-II specimen in Sbarbori et al. [2009] that was used for the repeated IZZI experiment was more SSD dominant, and hence provided Arai diagrams and Arai signatures like the specimen GA84.6c in this study (Figure 10g). The concave-up original Arai diagram is due to thermal alteration, which causes the TRM recording capability to increase over the $500^{\circ}-600^{\circ} \mathrm{C}$ temperature range (Figures $10 \mathrm{~g}$ and 12 in this study and Figures $8 \mathrm{a}$ and $8 \mathrm{~b}$ in [Sbarbori et al., 2009]), rather than the MD effect. This thermal alteration belongs to the hidden type that we discussed in section 5, which cannot be detected by pTRM checks.
[37] In this study, we do not consider possible anisotropy of the studied specimens from site GA-X. Anisotropy of samples can introduce slight uncertainties in the paleointensity estimations because the NRM and the laboratory-applied pTRM directions are not necessarily the same. However, any anisotropy effect can be readily compensated by applying the laboratory-applied tTRM in the same direction as the NRM before conducting the repeated BZF experiments. We will take this into account in future studies.

\section{Conclusions}

[38] The main magnetization carriers for the 24 studied samples from site GA-X are fine-grained low-titanium magnetite with various range of grain sizes (GA78 and GA 84 have more SSD grains; GA79 and GA 85 have more MD grains). The BZF protocol applied to the studied specimens is able to estimate paleointensites according to various methods, which provide self-consistency checks. This protocol can also be used in microwave and AF-based paleointensity techniques. For specimens dominated by MD magnetite grains, the standard pTRM checks can be affected by both thermophysicochemical alterations and non-linear (concave-up) Arai diagrams. Thus, pTRM checks are not always reliable indicators of alteration. The tTRM check, which detects the effects of alteration but not MD effects, is a more powerful and appropriate check than the pTRM check, although neither check is capable of detecting the hidden ( $T_{i}$ to $T_{i+1}$ type) thermophysicochemical alterations for both SSD or MD samples.

[39] Specimen pre-selection criteria based on rock magnetic properties developed in this study could be used to improve both paleointensity experiment success rate and quality of the results. Specimens that contain MD remanence carriers (low $\mathrm{M}_{\mathrm{r}} / \mathrm{M}_{\mathrm{S}}$ ratios) and that undergo minimal laboratory thermophysicochemical alteration during heating (reversible $\mathrm{J}_{\mathrm{s}}-\mathrm{T}$ curves and similar rock magnetic properties before and after heating) yield the most satisfactory Arai signature MD corrected paleointensity estimates. Rock magnetic criteria and results from our repeated BZF experiments suggest that MD-dominated samples provide more reliable and unbiased paleointensity estimates than more SSD-like samples, as long as the Arai signature MD correction is performed. Due to the fact that MD samples are much more common than SSD samples in nature, we suggest that our MD 
correction technique can be more generally used for paleointensity determinations.

[40] The final paleointensity estimate for site GA$\mathrm{X}$, which is based on 11 out of 24 specimens analyzed, is $4.23 \pm 1.29 \mu \mathrm{T}$ (mean \pm standard deviation; $4.16 \mu \mathrm{T}$ for median, Table 3), which happens to be almost identical to the median of the twopoint paleointensity estimates $(4.14 \mu \mathrm{T}$, Table 2$)$ from Kent et al. [2010].

\section{Acknowledgments}

[41] We thank Pierre Rochette for generously providing the Galapagos lava samples, Helene Collombat for organizing the sample collection with authorization of the National Park of the Galapagos Island, funded by INSU, and Lisa Tauxe for discussions on the properties of MD particles during paleointensity experiments. We thank the Associate Editor of our previous submission, Andrew Roberts, for his careful editing of the manuscript and two reviewers (Lisa Tauxe and Yongjae $\mathrm{Yu}$ ) for their constructive comments. We also thank G-cubed Editor James Tyburczy, Associate Editor Joshua Feinberg, and two anonymous reviewers of our current submission for their constructive comments, all of which have helped to improve this version of our paper. Finally, we thank the Wiley G-Cubed Production Editor and many others who have helped us to produce this article successfully. Research was supported by NSF grant EAR-1113569. LDEO contribution 7722.

\section{References}

Aitken, M. J., A. L. Allsop, G. D. Bussell, and M. B. Winter (1988), Determination of the intensity of the Earth's magnetic-field during archaeological times-Reliability of the Thellier technique, Rev. Geophys., 26, 3-12.

Ben-Yosef, E., H. Ron, L. Tauxe, A. Agnon, A. Genevey, T. E. Levy, U. Avner, and M. Najjar (2008), Application of copper slag in geomagnetic archaeointensity research, $J$. Geophys. Res., 113, B08101, doi:10.1029/2007JB005235.

Biggin, A. J., and H. N. Bohnel (2003), A method to reduce the curvature of Arai plots produced during Thellier palaeointensity experiments performed on multidomain grains, Geophys. J. Int., 155, F13-F19.

Biggin, A. J., and D. N. Thomas (2003), The application of acceptance criteria to results of Thellier palaeointensity experiments performed on samples with pseudo-single-domain-like characteristics, Phys. Earth Planet. Inter., 138, 279-287.

Butler, R. F., and S. K. Banerjee (1975), Theoretical singledomain grain size range in magnetite and titanomagnetite, $J$. Geophys. Res., 80, 4049-4058.

Coe, R. S. (1967), Paleo-intensities of the Earth's magnetic field determined from Tertiary and Quaternary rocks, $J$. Geophys. Res., 72, 3247-3262.

Day, R., M. Fuller, and V. A. Schmidt (1977), Hysteresis properties of titanomagnetites: Grain-size and compositional dependence, Phys. Earth Planet. Inter., 13, 260-267.

De Groot, L. V., E. A. Bakelaar, and M. J. Dekkers (2011), Detecting changes in magnetic domain configuration after heating using MFM imaging, Abstract GP23A-1023 pre- sented at 2011 Fall Meeting, AGU, San Francisco, Calif., 59 Dec.

Draeger, U., M. Prevot, T. Poidras, and J. Riisager (2006), Single-domain chemical, thermochemical and thermal remanences in a basaltic rock, Geophys. J. Int., 166, 12-32.

Dunlop, D. J., and O. Ozdemir (2000), Effect of grain size and domain state on thermal demagnetization tails, Geophys. Res. Lett., 27, 1311-1314.

Dunlop, D. J., and S. Xu (1994), Theory of partial thermoremanent magnetization in multidomain grains: 1. Repeated identical barriers to wall-motion (single microcoercivity), $J$. Geophys. Res., 99, 9005-9023.

Fabian, K. (2000), Acquisition of thermoremanent magnetization in weak magnetic fields, Geophys. J. Int., 142, 478-486.

Fabian, K. (2001), A theoretical treatment of paleointensity determination experiments on rocks containing pseudosingle or multi domain magnetic particles, Earth Planet. Sci. Lett., 188, 45-58.

Fabian, K. (2009), Thermochemical remanence acquisition in single-domain particle ensembles: A case for possible overestimation of the geomagnetic paleointensity, Geochem. Geophys. Geosyst., 10, Q06Z03, doi:10.1029/2009GC 002420.

Fabian, K., and V. P. Shcherbakov (2004), Domain state stabilization by iterated thermal magnetization processes, Geophys. J. Int., 159, 486-494.

Kent, D. V., H. Wang, and P. Rochette (2010), Equatorial paleosecular variation of the geomagnetic field from 0 to 3 Ma lavas from the Galapagos Islands, Phys. Earth Planet. Inter., 183, 404-412.

Kirschvink, J. L. (1980), The Least-squares line and plane and the analysis of palaeomagnetic data, Geophys. J. R. astr. SOC, 62, 699-718.

Leonhardt, R., C. Heunemann, and D. Krasa (2004), Analyzing absolute paleointensity determinations: Acceptance criteria and the software ThellierTool4.0, Geochem. Geophys. Geosyst., 5, Q12016, doi:10.1029/2004GC000807.

Levi, S. (1977), The effect of magnetite particle size on paleointensity determinations of the geomagnetic field, Phys. Earth Planet. Inter., 13, 245-259.

McClelland, E. (1996), Theory of CRM acquired by grain growth, and its implications for TRM discrimination and palaeointensity determination in igneous rocks, Geophys. $J$. Int., 126, 271-280.

Nagata, T., Y. Arai, and K. Momose (1963), Secular variation of the geomagnetic total force during the last 5000 years, $J$. Geophys. Res., 68, 5277-5281.

Neel, L. (1951), Le trainage magnetique, J. Phys. Radium, 12, 339-351.

Paterson, G. A. (2011), A simple test for the presence of multidomain behavior during paleointensity experiments, J. Geophys. Res., 116, B10104, doi :10.1029/2011JB008369.

Paterson, G. A. (2013), The effects of anisotropic and nonlinear thermoremanent magnetizations on Thellier-type paleointensity data, Geophys. J. Int., 193, 694-710.

Riisager, P., and J. Riisager (2001), Detecting multidomain magnetic grains in Thellier palaeointensity experiments, Phys. Earth Planet. Inter., 125, 111-117.

Rochette, P., F. Ben Atig, H. Collombat, D. Vandamme, and P. Vlag (1997), Low paleosecular variation at the equator: A paleomagnetic pilgrimage from Galapagos to Esterel with Allan Cox and Hans Zijderveld, Geol. Mijnbouw., 76, 9-19.

Sbarbori, E., L. Tauxe, A. Goguitchaichvili, J. Urrutia-Fucugauchi, and W. A. Bohrson (2009), Paleomagnetic behavior 
of volcanic rocks from Isla Socorro, Mexico, Earth Planets Space, 61, 191-204.

Shcherbakov, V. P., E. McClelland, and V. V. Shcherbakova (1993), A model of multidomain thermoremanent magnetization incorporating temperature-variable domain-structure, J. Geophys. Res., 98, 6201-6216.

Shcherbakova, V. V., V. P. Shcherbakov, and F. Heider (2000), Properties of partial thermoremanent magnetization in pseudosingle domain and multidomain magnetite grains, J. Geophys. Res., 105, 767-781.

Tauxe, L., and J. J. Love (2003), Paleointensity in Hawaiian Scientific Drilling Project Hole (HSDP2): Results from submarine basaltic glass, Geochem. Geophys. Geosyst., 4, 8702, doi: 10.1029/2001GC000276.

Tauxe, L., and H. Staudigel (2004), Strength of the geomagnetic field in the Cretaceous Normal Superchron: New data from submarine basaltic glass of the Troodos Ophiolite, Geochem. Geophys. Geosyst., 5, Q02H06, doi:10.1029/ 2003GC000635.

Thellier, E. (1938), Sur l'aimantation des terres cuites et ses applications geophysiques, Ann. I. P. G. Paris, 16, 157-302.

Thellier, E., and O. Thellier (1959), Sur l'intensite du champ magnetique terrestre dans le passe historique et geologique, Ann. Geophys., 15, 285-378.

Wilson, R. L. (1961), Palaeomagnetism in Northern Ireland, Part 1, The thermal demagnetization of natural magnetic moments in rocks, Geophys. J. Int., 5, 45-58.
Wilson, R. L. (1962), An instrument for measuring vector magnetization at high temperatures, Geophys. J. Int., 7, 125130.

Xu, S., and D. J. Dunlop (1994), Theory of partial thermoremanent magnetization in multidomain grains: 2. Effect of microcoercivity distribution and comparison with experiment, J. Geophys. Res., 99, 9025-9033.

Xu, S., and D. J. Dunlop (2004), Thellier paleointensity theory and experiments for multidomain grains, J. Geophys. Res., 109, B07103, doi:10.1029/2004JB003024.

Yamamoto, Y. (2006), Possible TCRM acquisition of the Kilauea 1960 lava, Hawaii: Failure of the Thellier paleointensity determination inferred from equilibrium temperature of the Fe-Ti oxide, Earth Planets Space, 58, 10331044.

Yu, Y. J., L. Tauxe, and A. Genevey (2004), Toward an optimal geomagnetic field intensity determination technique, Geochem. Geophys. Geosyst., 5, Q02H07, doi:10.1029/ 2003GC000630.

Yu, Y., and L. Tauxe (2005), Testing the IZZI protocol of geomagnetic field intensity determination, Geochem. Geophys. Geosyst., 6, Q05H17, doi:10.1029/2004GC 000840.

Zijderveld, J. D. A. (1967), A.C. demagnetization of rocks: Analysis of results, Methods in paleomagnetism, edited by D. W. Colllnson, K. M. Creer, and S. K. Runcorn, New York, Amsterdam, pp. 254-286. 